\title{
One-loop vertex correction in a plane wave
}

\author{
A. Di Piazza $\circledast^{1, *}$ and M. A. Lopez-Lopez $\oplus^{2,1, \uparrow}$ \\ ${ }^{1}$ Max Planck Institute for Nuclear Physics, Saupfercheckweg 1, D-69117 Heidelberg, Germany \\ ${ }^{2}$ Instituto de Física y Matemáticas, Universidad Michoacana de San Nicolás de Hidalgo, \\ Edificio C-3, Apdo. Postal 2-82, C.P. 58040 Morelia, Michoacán, Mexico
}

(Received 27 June 2020; accepted 24 September 2020; published 27 October 2020)

\begin{abstract}
We compute the general expression of the one-loop vertex correction in an arbitrary plane-wave background field for the case of two on-shell external electrons and an off-shell external photon. The properties of the vertex corrections under gauge transformations of the plane-wave background field and of the radiation field are studied. Concerning the divergences of the vertex correction, the infrared one is cured by assigning a finite mass to the photon, whereas the ultraviolet one is shown to be renormalized exactly as in vacuum. Finally, the corresponding expression of the vertex correction within the locally constant crossed field approximation is also derived and the high-field asymptotic is shown to scale according to the Ritus-Narozhny conjecture, although the terms featuring the leading-order dependence on the field are found not to contribute to physical transition amplitudes.
\end{abstract}

DOI: 10.1103/PhysRevD.102.076018

\section{INTRODUCTION}

The predictions of QED agree with experiments with impressive accuracy (see, e.g., Refs. [1,2]). The great success of QED has called for testing this theory under more extreme conditions as, for example, those provided by intense background electromagnetic fields. An electromagnetic field is denoted as "intense" in the realm of QED if it is of the order of the so-called "critical" field of QED: $F_{c r}=m^{2} /|e|=1.3 \times 10^{16} \mathrm{~V} / \mathrm{cm}=4.4 \times 10^{13} \mathrm{G} \quad$ (from now on we employ units with $\epsilon_{0}=\hbar=c=1$ and $m$ and $e<0$ denote the electron mass and charge, respectively) [3-5]. Importantly, the presence of intense background electromagnetic fields allows for testing QED on a sector where nonlinear effects with respect to the background field strongly affect physical processes and the dynamics of charged particles. The theoretical and experimental investigation of the nonlinear sector of QED can serve as an additional ground test of the theory, as compared to the more traditional one successfully scrutinized via conventional accelerators.

High-power optical lasers are becoming a suitable tool to test QED at critical field strengths, which correspond to laser intensities of the order of $10^{29} \mathrm{~W} / \mathrm{cm}^{2}$. In fact, although

\footnotetext{
dipiazza@mpi-hd.mpg.de

†misha.lopez@umich.mx
}

Published by the American Physical Society under the terms of the Creative Commons Attribution 4.0 International license. Further distribution of this work must maintain attribution to the author(s) and the published article's title, journal citation, and DOI. Funded by SCOAP ${ }^{3}$. available lasers have reached peak intensities $I_{0}$ of the order of $5.5 \times 10^{22} \mathrm{~W} / \mathrm{cm}^{2}[6]$ and upcoming facilities aim at $I_{0} \sim 10^{23}-10^{24} \mathrm{~W} / \mathrm{cm}^{2} \quad[7-10]$, physical observables depend only on Lorentz-invariant quantities, such as for processes initiated by a single electron/positron, the quantum nonlinearity parameter $\chi_{0}=\sqrt{-\left(p_{\mu} F_{0}^{\mu \nu}\right)^{2}} / m F_{c r}$ [11-16], where $F_{0}^{\mu \nu}$ characterizes the amplitude of the laser field and $p^{\mu}$ is the initial four-momentum of the electron/positron [the metric tensor $\eta^{\mu \nu}=\operatorname{diag}(+1,-1,-1,-1)$ is employed throughout]. Since the parameter $\chi_{0}$ numerically corresponds to the amplitude of the electric field of the laser in the rest frame of the charge in units of $F_{c r}$, one can see that the strong-field QED regime $\chi_{0} \gtrsim 1$ can be entered already at intensities of the order of $10^{23} \mathrm{~W} / \mathrm{cm}^{2}$, if the laser field counterpropagates with respect to an electron/positron with energy of the order of $500 \mathrm{MeV}$.

In order to test QED in the strong-field regime by means of intense optical fields, it is essential that both experiments and theoretical predictions are correspondingly accurate. However, as it is understandable, first experiments in this regime have so far been designed especially to show the occurrence of phenomena such as nonlinear Compton scattering [17], nonlinear Breit-Wheeler pair production $[18,19]$, and radiation reaction [20,21], without aiming at obtaining high-accuracy results. Correspondingly, on the theory side, the basic strong-field QED processes such as nonlinear Compton scattering [12,22-49] and nonlinear Breit-Wheeler pair production [14,23,48,50-62] have been studied in detail at tree level by approximating the laser field as a plane wave (see also the reviews [11-15]). However, even the one-loop radiative corrections to the 
probabilities of these processes have never been computed. The reason is that calculations including the effects of the external laser field exactly are significantly more complex than the corresponding calculations in vacuum. The standard technique, in fact, is to work within the so-called Furry picture [63], where the electron-positron field is quantized in the presence of the background field [3,4]. This requires that the Dirac equation can be solved analytically in the presence of the background field, which has been achieved in Ref. [64] in the case of a plane wave (see also Ref. [3]), the corresponding states being known as Volkov states. An alternative, equivalent technique is the so-called operator technique, first proposed by Schwinger [65] and then developed for the case of a background plane wave [66-71], which does not require the explicit solution of the Dirac equation in the plane-wave field.

Going back to the radiative corrections, a systematic study has been carried out only in the special case of a zerofrequency plane wave or a constant crossed field, i.e., a constant and uniform electromagnetic field with electric and magnetic fields having the same amplitude and being perpendicular to each other, from the early works of Ritus and Narozhny [72-79] to the more recent one [80] (see also Ref. [81] and the reviews in Refs. [82,83]), where higher-loop Feynman diagrams have been evaluated. In particular, in Ref. [72] the one-loop corrections to the electron and photon mass were studied, in Ref. [73] the general structures of the mass operator and of the polarization operator were investigated, in Ref. [74] the two-loop diagram corresponding to the polarization operator inserted into the mass operator was computed, whereas the remaining two proper one-particle irreducible diagrams contributing to the two-loop mass operator containing two internal photon lines were evaluated in Ref. [75]. Three-loop diagrams contributing to the mass operator and to the polarization operator (not involving the vertex correction) were computed in Ref. [76] together with their large-field asymptotics, a study which was continued in Ref. [77]. Finally, the one-loop vertex correction was evaluated in Refs. [78,79]. However, so far, in the case of a general plane wave with an arbitrary polarization and shape, only the one-loop mass operator (see Fig. 1) and the one-loop polarization operator (see Fig. 2) have been computed in Ref. [66] and in Refs. [67,84], respectively (see also Ref. [85] for an alternative derivation of the polarization operator). The one-loop vertex correction in a general plane wave (see Fig. 3) has never been evaluated, whereas, as we have mentioned, the corresponding quantity in a constant crossed field was computed in Refs. [78,79]. The purpose of the present paper is to fill this gap and, indeed, to compute the one-loop vertex correction in an arbitrary plane wave for the case of two on-shell external electrons and an off-shell external photon. It is worth mentioning here that the computation of the vertex-correction function is not only important to evaluate the leading-order radiative

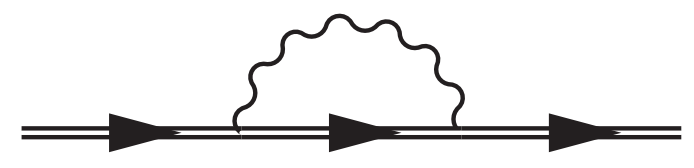

FIG. 1. The one-loop mass operator in an intense plane wave. The double lines represent exact electron states and propagator in a plane wave (Volkov states and propagator, respectively) [3].

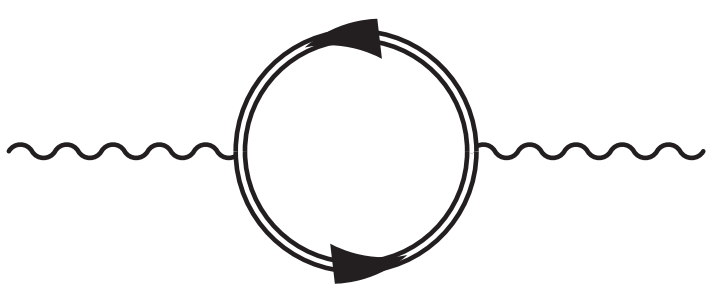

FIG. 2. The one-loop polarization operator in an intense plane wave. The double lines represent exact electron propagators in a plane wave (Volkov propagators) [3].

corrections of strong-field QED processes. There is also a more fundamental reason related to the so-called RitusNarozhny conjecture [72,76-79] about the high-field behavior of radiative corrections in strong-field QED in a constant crossed field. As we have mentioned, a constant crossed field is a constant and uniform electromagnetic field $F_{0}^{\mu \nu}=\left(\boldsymbol{E}_{0}, \boldsymbol{B}_{0}\right)$ such that the two field Lorentz invariants $\boldsymbol{E}_{0}^{2}-\boldsymbol{B}_{0}^{2}$ and $\boldsymbol{E}_{0} \cdot \boldsymbol{B}_{0}$ vanish. Now, in a constant crossed field radiative corrections depend only on the Lorentz- and gauge-invariant parameter $\chi_{0}$ introduced above. The Ritus-Narozhny conjecture states that at $\chi_{0} \gg 1$ the effective coupling of QED in a constant crossed field scales as $\alpha \chi_{0}^{2 / 3}$, where $\alpha=e^{2} / 4 \pi \approx 1 / 137$ is the finestructure constant, which implies an asymptotic highenergy behavior of strong-field QED in a constant crossed

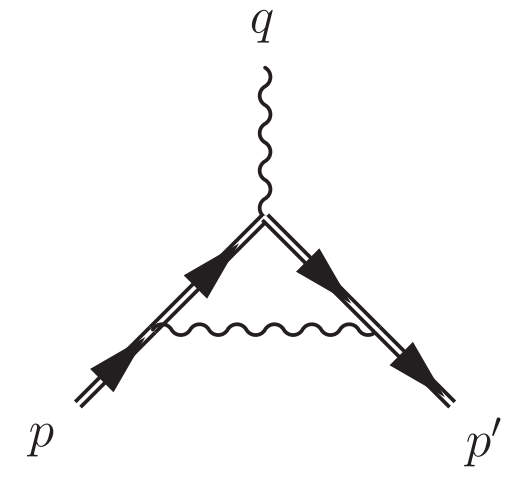

FIG. 3. The one-loop Feynman diagram corresponding to the vertex correction. The double lines represent exact electron states and propagator in a plane wave (Volkov states and propagator, respectively) [3]. 
field qualitatively different from the logarithmic one of QED in vacuum [3,86-88]. The physical relevance of the Ritus-Narozhny conjecture is broadened by the so-called locally constant field approximation (LCFA), stating that in the limit of low-frequency plane waves the probabilities of QED processes reduce to the corresponding probabilities in a constant crossed field averaged over the phase-dependent plane-wave profile [12]. In Ref. [89] we have investigated the one-loop mass and polarization operator to show that, if one first performs in the general expression of these quantities the high-energy limit, one indeed recovers the typical logarithmic behavior of QED as in vacuum (see also Ref. [90]). Below, we will also investigate the vertex correction within the LCFA, whereas the high-energy asymptotic will be presented elsewhere.

The paper is organized as follows: In Sec. II we introduce the basic notation of the paper. In Sec. III the general form of the vertex-correction function is derived by means of the operator technique. In Sec. IV the properties of the vertexcorrection function under gauge transformations of the radiation field and of the plane-wave background field are studied. In Sec. V we show how to regularize and renormalize the vertex-correction function in the ultraviolet limit of the four-momentum circulating in the loop. The expression of the vertex-correction function within the LCFA is derived in Sec. VI, and, finally, the main conclusions of the paper are reported in Sec. VII. The Appendix contains some technical considerations on a component of the vertex-correction function.

\section{NOTATION}

The notation employed below is the same as in Ref. [71] but it is convenient to report here the main definitions. As we have mentioned in the Introduction, the present paper focuses on studying radiative corrections in a general plane-wave field. The latter is described by the four-vector potential $A^{\mu}(\phi)$, which only depends on the light-cone time $\phi=t-\boldsymbol{n} \cdot \boldsymbol{x}$. Here, the unit vector $\boldsymbol{n}$ defines the propagation direction of the plane wave, which can be used to introduce two useful four-dimensional quantities: $n^{\mu}=$ $(1, \boldsymbol{n})$ and $\tilde{n}^{\mu}=(1,-\boldsymbol{n}) / 2$ [note that $\phi=(n x)$ ]. Assuming obvious differential properties of the four-vector potential $A^{\mu}(\phi)$ and its derivatives, it is clear that it is a solution of the free wave equation $\square A^{\mu}=0$, where $\square=\partial_{\nu} \partial^{\nu}$, and it is assumed to fulfill the Lorenz-gauge condition $\partial_{\mu} A^{\mu}=0$, with the additional constraint $A^{0}(\phi)=0$. Thus, if we represent $A^{\mu}(\phi)$ in the form $A^{\mu}(\phi)=(0, \boldsymbol{A}(\phi))$, then the Lorenz-gauge condition implies $\boldsymbol{n} \cdot \boldsymbol{A}^{\prime}(\phi)=0$, with the prime in a function of $\phi$ indicating its derivative with respect to $\phi$. If we make the additional assumption that $\boldsymbol{A}(\phi)$ vanishes for $\phi \rightarrow \pm \infty$, the equality $\boldsymbol{n} \cdot \boldsymbol{A}^{\prime}(\phi)=0$ implies that $\boldsymbol{n} \cdot \boldsymbol{A}(\phi)=0$. By introducing two four-vectors $a_{j}^{\mu}=\left(0, \boldsymbol{a}_{j}\right)$, with $j=1,2$, such that $\left(n a_{j}\right)=-\boldsymbol{n} \cdot \boldsymbol{a}_{j}=0$ and $\left(a_{i} a_{j}\right)=-\boldsymbol{a}_{i} \cdot \boldsymbol{a}_{j}=-\delta_{i j}$, the most general form of the vector potential $\boldsymbol{A}(\phi)$ reads $\boldsymbol{A}(\phi)=\psi_{1}(\phi) \boldsymbol{a}_{1}+\psi_{2}(\phi) \boldsymbol{a}_{2}$, where the two functions $\psi_{j}(\phi)$ are arbitrary provided that they vanish for $\phi \rightarrow \pm \infty$ and they feature the differential properties mentioned above when the four-vector potential $A^{\mu}(\phi)$ was introduced. The field tensor $F^{\mu \nu}(\phi)=$ $\partial^{\mu} A^{\nu}(\phi)-\partial^{\nu} A^{\mu}(\phi)$ of the plane wave is given by $F^{\mu \nu}(\phi)=n^{\mu} A^{\prime \nu}(\phi)-n^{\nu} A^{\prime \mu}(\phi)$, and below we will also use its integral $\mathscr{F}^{\mu \nu}(\phi)=\int_{-\infty}^{\phi} d \phi^{\prime} F^{\mu \nu}\left(\phi^{\prime}\right)=n^{\mu} A^{\nu}(\phi)-$ $n^{\nu} A^{\mu}(\phi)$ [note that the tensor $\mathscr{F}^{\mu \nu}(\phi)$ is gauge invariant].

The four-dimensional quantities $n^{\mu}, \tilde{n}^{\mu}$, and $a_{j}^{\mu}$ fulfill the completeness relation: $\eta^{\mu \nu}=n^{\mu} \tilde{n}^{\nu}+\tilde{n}^{\mu} n^{\nu}-a_{1}^{\mu} a_{1}^{\nu}-a_{2}^{\mu} a_{2}^{\nu}$ [note that $(n \tilde{n})=1$ and $\left(\tilde{n} a_{j}\right)=0$ ]. Below, we will refer to the longitudinal ( $n$ ) direction as the direction along $\boldsymbol{n}$ and to the transverse $(\perp)$ plane as the plane spanned by the two perpendicular unit vectors $\boldsymbol{a}_{j}$. In this respect, together with the light-cone time $\phi=t-x_{n}$, with $x_{n}=\boldsymbol{n} \cdot \boldsymbol{x}$, we also introduce the remaining three light-cone coordinates $T=(\tilde{n} x)=\left(t+x_{n}\right) / 2$, and $\boldsymbol{x}_{\perp}=\left(x_{\perp, 1}, x_{\perp, 2}\right)=-\left(\left(x a_{1}\right)\right.$, $\left.\left(x a_{2}\right)\right)=\left(\boldsymbol{x} \cdot \boldsymbol{a}_{1}, \boldsymbol{x} \cdot \boldsymbol{a}_{2}\right)$. Analogously, the light-cone coordinates of an arbitrary four-vector $v^{\mu}=\left(v^{0}, v\right)$ will be indicated as $v_{-}=(n v)=v^{0}-v_{n}$, with $v_{n}=\boldsymbol{n} \cdot \boldsymbol{v}, v_{+}=$ $(\tilde{n} v)=\left(v^{0}+v_{n}\right) / 2, \quad$ and $\quad \boldsymbol{v}_{\perp}=\left(v_{\perp, 1}, v_{\perp, 2}\right)=-\left(\left(v a_{1}\right)\right.$, $\left.\left(v a_{2}\right)\right)=\left(\boldsymbol{v} \cdot \boldsymbol{a}_{1}, \boldsymbol{v} \cdot \boldsymbol{a}_{2}\right)$. Since we will employ the operator technique, it is convenient to also introduce the momenta operators $P_{\phi}=-i \partial_{\phi}=-(\tilde{n} P)=-\left(i \partial_{t}-i \partial_{x_{n}}\right) / 2, P_{T}=$ $-i \partial_{T}=-(n P)=-\left(i \partial_{t}+i \partial_{x_{n}}\right)$, and $\boldsymbol{P}_{\perp}=\left(P_{\perp, 1}, P_{\perp, 2}\right)=$ $-i\left(\boldsymbol{a}_{1} \cdot \nabla, \boldsymbol{a}_{2} \cdot \nabla\right)$. These operators are the momenta conjugated to the light-cone coordinates in the sense that the commutator between the operator corresponding to each light-cone coordinate and the associated momentum operator is equal to the imaginary unit (all other possible commutators vanish): $\left[\phi, P_{\phi}\right]=\left[T, P_{T}\right]=i$ and $\left[X_{\perp, j}, P_{\perp, k}\right]=i \delta_{j k}$, which are equivalent to the commutation relations $\left[X^{\mu}, P^{\nu}\right]=-i \eta^{\mu \nu}$, with $P^{\mu}=i \partial^{\mu}$.

The commutation relations $\left[X^{\mu}, P^{\nu}\right]=-i \eta^{\mu \nu}$ imply that $\left[P^{\mu}, f(X)\right]=i \partial_{X}^{\mu} f(X)$, where $f(X)$ is an arbitrary function of the four-position operator that can be expanded in Taylor series and $\partial_{X}^{\mu}=\partial / \partial X_{\mu}$. Analogously, it can easily be shown that $\exp [i f(X)] P^{\mu} \exp [-i f(X)]=P^{\mu}+\partial^{\mu} f(X)$ and then formally that $\exp [i f(X)] g(P) \exp [-i f(X)]=g(P+\partial f(X))$, where $g(P)$ is a function of the four-momentum that can be expanded in Taylor series [this identity has to be intended to apply to the Taylor series expansion of the function $g(P)$ ]. The same commutation relations imply that $\exp [i g(P)] X^{\mu} \exp [-i g(P)]=X^{\mu}-\partial_{P}^{\mu} g(P)$ and that $\exp [i g(P)] f(X) \exp [-i g(P)]=f\left(X-\partial_{P} g(P)\right)$, where $\partial_{P}^{\mu}=$ $\partial / \partial P_{\mu}$ [as above, this identity has to be intended to apply to the Taylor series expansion of the function $f(X)$ ]. In particular, we will consider the case where the functions in the exponents are linear either in $X^{\mu}$ or in $P^{\mu}$ :

$$
\begin{aligned}
& \exp (i(X q)) g(P) \exp (-i(X q))=g(P+q), \\
& \exp (i(P y)) f(X) \exp (-i(P y))=f(X-y),
\end{aligned}
$$


where $q^{\mu}$ and $y^{\mu}$ are constant four-vectors.

In addition, the commutation relations $\left[\phi, P_{\phi}\right]=$ $\left[T, P_{T}\right]=i$ imply, in particular, the identities

$$
\begin{aligned}
& \exp (i a \phi) \tilde{g}\left(P_{\phi}\right) \exp (-i a \phi)=\tilde{g}\left(P_{\phi}-a\right), \\
& \exp \left(i b P_{T}\right) \tilde{f}(T) \exp \left(-i b P_{T}\right)=\tilde{f}(T+b),
\end{aligned}
$$

with $a$ and $b$ being two constants and $\tilde{f}(T)$ and $\tilde{g}\left(P_{\phi}\right)$ being two arbitrary functions, which we will use below.

Note that if $|x\rangle(|p\rangle)$ is the eigenstate of the four-position (four-momentum) operator $X^{\mu}\left(P^{\mu}=i \partial^{\mu}\right)$ with eigenvalue $x^{\mu}\left(p^{\mu}\right)$, i.e., $X^{\mu}|x\rangle=x^{\mu}|x\rangle \quad\left(P^{\mu}|p\rangle=p^{\mu}|p\rangle\right)$, then, by normalizing the eigenstates $|x\rangle(|p\rangle)$ such that $\langle x \mid y\rangle=$ $\delta^{(4)}(x-y) \quad\left[\langle p \mid q\rangle=(2 \pi)^{4} \delta^{(4)}(p-q)\right], \quad$ it $\quad$ is $\quad\langle x \mid p\rangle=$ $\exp (-i(p x))=\exp \left[-i\left(p_{+} \phi+p_{-} T-\boldsymbol{p}_{\perp} \cdot \boldsymbol{x}_{\perp}\right)\right]$ and $P_{\phi}|p\rangle=$ $-p_{+}|p\rangle, P_{T}|p\rangle=-p_{-}|p\rangle$, as well as $\boldsymbol{P}_{\perp}|p\rangle=\boldsymbol{p}_{\perp}|p\rangle$. Also, the operator completeness relations hold:

$$
\begin{gathered}
\int d^{4} x|x\rangle\langle x|=1, \\
\int \frac{d^{4} p}{(2 \pi)^{4}}|p\rangle\langle p|=1 .
\end{gathered}
$$

The Volkov states are the exact, analytical solutions of the Dirac equation in a plane wave $[64,3]$. The positiveenergy Volkov states $U_{s}(p, x)$ can be classified by means of the asymptotic momentum quantum numbers $\boldsymbol{p}$ (and then the energy $\varepsilon=\sqrt{m^{2}+\boldsymbol{p}^{2}}$ ) and of the asymptotic spin quantum number $s=1,2$ in the remote past, i.e., for $t \rightarrow-\infty$ (for notational simplicity, we have indicated the functional dependence on the four components of the electron four-momentum $p^{\mu}=(\varepsilon, \boldsymbol{p})$, although the energy is a function of the linear momentum). Following the general notation in Ref. [3], these states can be written as $U_{s}(p, x)=E(p, x) u_{s}(p)$, where

$E(p, x)=\left[1+\frac{e \hat{n} \hat{A}(\phi)}{2 p_{-}}\right] \mathrm{e}^{i\left\{-(p x)-\int_{-\infty}^{\phi} d \varphi\left[\frac{[(p A(\varphi))}{p_{-}}-\frac{e^{2} A^{2}(\varphi)}{2 p_{-}}\right]\right\}}$,

and where $u_{s}(p)$ are the free, positive-energy spinors normalized as $u_{s}^{\dagger}(p) u_{s^{\prime}}(p)=2 \varepsilon \delta_{s s^{\prime}}$ [3]. In Eq. (7) we have introduced the notation $\hat{v}=\gamma^{\mu} v_{\mu}$ for a generic four-vector $v^{\mu}$, with $\gamma^{\mu}$ being the Dirac matrices, which satisfy the anticommutation relations $\left\{\gamma^{\mu}, \gamma^{\nu}\right\}=2 \eta^{\mu \nu}$ [3].

The electron Green's function $G\left(x, x^{\prime}\right)$ in the general plane-wave background electromagnetic field described by the four-vector potential $A^{\mu}(\phi)$ is defined by the equation

$$
\left\{\gamma^{\mu}\left[i \partial_{\mu}-e A_{\mu}(\phi)\right]-m\right\} G\left(x, x^{\prime}\right)=\delta^{(4)}\left(x-x^{\prime}\right) .
$$

In order to uniquely identify the Green's function, boundary conditions also have to be specified. Here, we always assume the Feynman prescription corresponding to the shift $m \rightarrow m-i 0$ [3]. Within the operator technique the operator $G$ corresponding to the Green's function $G\left(x, x^{\prime}\right)$ is defined via the equation $G\left(x, x^{\prime}\right)=\left\langle x|G| x^{\prime}\right\rangle$, i.e., as

$$
G=\frac{1}{\hat{\Pi}-m+i 0},
$$

where $\Pi^{\mu}=P^{\mu}-e A^{\mu}(\Phi)$. Now, we have explicitly shown in Ref. [71] (see also Refs. [66-68]) that the operator $G$ can be written in the form

$$
\begin{aligned}
G= & (\hat{\Pi}+m) \frac{1}{\hat{\Pi}^{2}-m^{2}+i 0}=(\hat{\Pi}+m)(-i) \\
& \times \int_{0}^{\infty} d s e^{-i m^{2} s} e^{2 i s P_{T} P_{\phi}} e^{-i \int_{0}^{s} d s^{\prime}\left[\boldsymbol{P}_{\perp}-e \boldsymbol{A}_{\perp}\left(\Phi-2 s^{\prime} P_{T}\right)\right]^{2}} \\
& \times\left\{1-\frac{e}{2 P_{T}} \hat{n}\left[\hat{A}\left(\Phi-2 s P_{T}\right)-\hat{A}(\Phi)\right]\right\}
\end{aligned}
$$

where the prescription $m^{2} \rightarrow m^{2}-i 0$ is understood. Below, we will also need the equivalent expression

$$
\begin{aligned}
G= & \frac{1}{\hat{\Pi}^{2}-m^{2}+i 0}(\hat{\Pi}+m)=(-i) \int_{0}^{\infty} d s e^{-i m^{2} s} \\
& \times\left\{1+\frac{e}{2 P_{T}} \hat{n}\left[\hat{A}\left(\Phi+2 s P_{T}\right)-\hat{A}(\Phi)\right]\right\} \\
& \times e^{-i \int_{0}^{s} d s^{\prime}\left[\boldsymbol{P}_{\perp}-e \boldsymbol{A}_{\perp}\left(\Phi+2 s^{\prime} P_{T}\right)\right]^{2}} e^{2 i s P_{T} P_{\phi}(\hat{\Pi}+m) .}
\end{aligned}
$$

\section{GENERAL EXPRESSION OF THE ONE-LOOP VERTEX CORRECTION}

The one-loop vertex correction corresponds to the Feynman diagram in Fig. 3, where we have implicitly assumed that the photon four-momentum $q^{\mu}$ is outgoing. Note that the two external electron lines correspond to real electrons; i.e., the four-momenta $p^{\mu}$ and $p^{\prime \mu}$ are on-shell $\left(p^{2}=p^{2}=m^{2}\right)$, whereas at the moment we make no assumptions about the outgoing photon, i.e., in particular, $q^{2} \neq 0$. If we denote by $s\left(s^{\prime}\right)$ the spin quantum number of the incoming (outgoing) electron and by $l$ the polarization quantum number of the outgoing photon, the amplitude $-i e \Gamma_{s, s^{\prime}, l}\left(p, p^{\prime}, q\right)$ corresponding to the Feynman diagram in Fig. 3 can be written as

$$
\begin{aligned}
& -i e \Gamma_{s, s^{\prime}, l}\left(p, p^{\prime}, q\right) \\
& =-e^{3} \int d^{4} x d^{4} y d^{4} z \bar{U}_{s^{\prime}}\left(p^{\prime}, y\right) \gamma^{\lambda} G(y, z) \hat{e}_{l}^{*}(q) \\
& \quad \times e^{i(q z)} G(z, x) \gamma^{\nu} U_{s}(p, x) D_{\lambda \nu}(x-y),
\end{aligned}
$$

where $e_{l}^{\mu}(q)$ is the polarization four-vector of the outgoing photon. Here, we have introduced the photon propagator $D^{\lambda \nu}(x)$, and we work in the Lorenz gauge such that 


$$
D^{\lambda \nu}(x)=\int \frac{d^{4} k}{(2 \pi)^{4}} \frac{\eta^{\lambda \nu}}{k^{2}-\kappa^{2}+i 0} e^{-i(k x)}
$$

where $\kappa^{2}$ is the square of a fictitious photon mass, which has been introduced to avoid infrared divergences.

By using the completeness relation in Eq. (5) and the translation properties in Eq. (1), the amplitude can be written in the semioperator form as

$$
\begin{aligned}
-i e \Gamma_{s, s^{\prime}, l}\left(p, p^{\prime}, q\right)= & -e^{3} \int d^{4} x \int \frac{d^{4} k}{(2 \pi)^{4}} \frac{1}{k^{2}-\kappa^{2}+i 0} \bar{U}_{s^{\prime}}\left(p^{\prime}, x\right) e^{i(k x)} \gamma^{\lambda} G e^{i(q x)} \hat{e}_{l}^{*}(q) G e^{-i(k x)} \gamma_{\lambda} U_{s}(p, x) \\
= & -e^{3} \int d^{4} x \int \frac{d^{4} k}{(2 \pi)^{4}} \frac{1}{k^{2}-\kappa^{2}+i 0} \\
& \times \bar{U}_{s^{\prime}}\left(p^{\prime}, x\right) \gamma^{\lambda} \frac{1}{\hat{\Pi}(\phi)+\hat{k}-m+i 0} e^{i(q x)} \hat{e}_{l}^{*}(q) \frac{1}{\hat{\Pi}(\phi)+\hat{k}-m+i 0} \gamma_{\lambda} U_{s}(p, x) \\
= & -e^{3} \int d^{4} x \int \frac{d^{4} k}{(2 \pi)^{4}} \frac{1}{k^{2}-\kappa^{2}+i 0} \\
& \times \bar{U}_{s^{\prime}}\left(p^{\prime}, x\right) \gamma^{\lambda}[\hat{\Pi}(\phi)+\hat{k}+m] \frac{1}{[\hat{\Pi}(\phi)+\hat{k}]^{2}-m^{2}+i 0} e^{i(q x)} \hat{e}_{l}^{*}(q) \\
& \times \frac{1}{[\hat{\Pi}(\phi)+\hat{k}]^{2}-m^{2}+i 0}[\hat{\Pi}(\phi)+\hat{k}+m] \gamma_{\lambda} U_{s}(p, x),
\end{aligned}
$$

where $\Pi^{\mu}(\phi)=i \partial^{\mu}-e A^{\mu}(\phi)$. By using the fact that $[\hat{\Pi}(\phi)-m] U_{s}(p, x)=[\hat{\Pi}(\phi)-m] U_{s^{\prime}}\left(p^{\prime}, x\right)=0$, we obtain

$$
\begin{aligned}
-i e \Gamma_{s, s^{\prime}, l}\left(p, p^{\prime}, q\right)= & -e^{3} \int d^{4} x \int \frac{d^{4} k}{(2 \pi)^{4}} \frac{1}{k^{2}-\kappa^{2}+i 0} \\
& \times \bar{U}_{s^{\prime}}\left(p^{\prime}, x\right)\left[2 \Pi^{\lambda}(\phi)+\gamma^{\lambda} \hat{k}\right] \frac{1}{[\hat{\Pi}(\phi)+\hat{k}]^{2}-m^{2}+i 0} e^{i(q x)} \hat{e}_{l}^{*}(q) \\
& \times \frac{1}{[\hat{\Pi}(\phi)+\hat{k}]^{2}-m^{2}+i 0}\left[2 \Pi_{\lambda}(\phi)+\hat{k} \gamma_{\lambda}\right] U_{s}(p, x) .
\end{aligned}
$$

Now, we notice that [see Eq. (7)]

$$
\Pi^{\lambda}(\phi) U_{s}(p, x)=\left[\pi_{p}^{\lambda}(\phi)+i \frac{e \hat{n} \hat{A}^{\prime}(\phi)}{2 p_{-}} n^{\lambda}\right] U_{s}(p, x)
$$

where

$$
\pi_{p}^{\lambda}(\phi)=p^{\lambda}-e A^{\lambda}(\phi)+\frac{e(p A(\phi))}{p_{-}} n^{\lambda}-\frac{e^{2} A^{2}(\phi)}{2 p_{-}} n^{\lambda}
$$

is the classical kinetic four-momentum of an electron in the plane wave $A^{\mu}(\phi)$, with $\lim _{\phi \rightarrow \pm \infty} \pi_{p}^{\lambda}(\phi)=p^{\lambda}$. The kinetic fourmomentum $\pi_{p}^{\lambda}(\phi)$ is clearly a gauge-invariant four-vector and, by using the tensor $\mathscr{F}^{\mu \nu}(\phi)$ (see Sec. II), it can be written in the manifestly gauge-invariant form as

$$
\pi_{p}^{\lambda}(\phi)=p^{\lambda}-\frac{e p_{\mu} \mathscr{F}^{\mu \lambda}(\phi)}{p_{-}}+\frac{e^{2} p_{\mu} \mathscr{F}^{\mu \rho}(\phi) \mathscr{F}_{\rho \nu}(\phi) p^{\nu}}{2 p_{-}^{3}} n^{\lambda}
$$

In this way, the quantity $-i e \Gamma_{s, s^{\prime}, l}\left(p, p^{\prime}, q\right)$ can be written as 


$$
\begin{aligned}
-i e \Gamma_{s, s^{\prime}, l}\left(p, p^{\prime}, q\right)= & -e^{3} \int d^{4} x \int \frac{d^{4} k}{(2 \pi)^{4}} \frac{1}{k^{2}-\kappa^{2}+i 0} \\
& \times \bar{U}_{s^{\prime}}\left(p^{\prime}, x\right)\left[2 \pi_{p^{\prime}}^{\lambda}(\phi)+i \frac{e \hat{n} \hat{A}^{\prime}(\phi)}{p_{-}^{\prime}} n^{\lambda}+\gamma^{\lambda} \hat{k}\right] \frac{1}{[\hat{\Pi}(\phi)+\hat{k}]^{2}-m^{2}+i 0} e^{i(q x)} \hat{e}_{l}^{*}(q) \\
& \times \frac{1}{[\hat{\Pi}(\phi)+\hat{k}]^{2}-m^{2}+i 0}\left[2 \pi_{p, \lambda}(\phi)+i \frac{e \hat{n} \hat{A}^{\prime}(\phi)}{p_{-}} n_{\lambda}+\hat{k} \gamma_{\lambda}\right] U_{s}(p, x) .
\end{aligned}
$$

At this point, it is convenient to use the representations in Eq. (10) and in Eq. (11) for the second and the first square Volkov propagator $1 /\left\{[\hat{\Pi}(\phi)+\hat{k}]^{2}-m^{2}+i 0\right\}$, respectively:

$$
\begin{aligned}
-i e \Gamma_{s, s^{\prime}, l}\left(p, p^{\prime}, q\right)= & e^{3} \int d^{4} x \int \frac{d^{4} k}{(2 \pi)^{4}} \int_{0}^{\infty} d s \int_{0}^{\infty} d u \frac{e^{i(q x)}}{k^{2}-\kappa^{2}+i 0} \\
& \times \bar{U}_{s^{\prime}}\left(p^{\prime}, x\right)\left[2 \pi_{p^{\prime}}^{\lambda}(\phi)+i \frac{e \hat{n} \hat{A}^{\prime}(\phi)}{p_{-}^{\prime}} n^{\lambda}+\gamma^{\lambda} \hat{k}\right] e^{-i m^{2} s} e^{-2 i s\left(p_{-}-q_{-}+k_{-}\right)\left(P_{\phi^{-}}-k_{+}+q_{+}\right)} \\
& \times e^{-i \int_{0}^{s} d s^{\prime}\left[\boldsymbol{p}_{\perp}-q_{\perp}+\boldsymbol{k}_{\perp}-e \boldsymbol{A}_{\perp}\left(\phi+2 s^{\prime}\left(p_{-}-q_{-}+k_{-}\right)\right)\right]^{2}}\left\{1+\frac{e \hat{n}\left[\hat{A}\left(\phi+2 s\left(p_{-}-q_{-}+k_{-}\right)\right)-\hat{A}(\phi)\right]}{2\left(p_{-}-q_{-}+k_{-}\right)}\right\} \\
& \times \hat{e}_{l}^{*}(q) e^{-i m^{2} u}\left\{1-\frac{e \hat{n}\left[\hat{A}\left(\phi-2 u\left(p_{-}+k_{-}\right)\right)-\hat{A}(\phi)\right]}{2\left(p_{-}+k_{-}\right)}\right\} e^{\left.-i \int_{0}^{u} d u^{\prime} \boldsymbol{p}_{\perp}+\boldsymbol{k}_{\perp}-e \boldsymbol{A}_{\perp}\left(\phi-2 u^{\prime}\left(p_{-}+k_{-}\right)\right)\right]^{2}} \\
& \times e^{-2 i u\left(p_{-}+k_{-}\right)\left(P_{\phi}-k_{+}\right)}\left[2 \pi_{p, \lambda}(\phi)+i \frac{e \hat{n} \hat{A}^{\prime}(\phi)}{p_{-}} n_{\lambda}+\hat{k} \gamma_{\lambda}\right] U_{s}(p, x),
\end{aligned}
$$

where we have exploited the fact that Volkov states are eigenstates of the operators $P_{T}$ and $\boldsymbol{P}_{\perp}$. Indeed, the only operator remaining in this equation is $P_{\phi}$. Now, we use the translation property in Eq. (2) and, analogously to the vacuum case, we write the amplitude $-i e \Gamma_{s, s^{\prime}, l}\left(p, p^{\prime}, q\right)$ in the form

$$
-i e \Gamma_{s, s^{\prime}, l}\left(p, p^{\prime}, q\right)=-i e \int d^{4} x e^{i(q x)} \bar{U}_{s^{\prime}}\left(p^{\prime}, x\right) \Gamma^{\mu}\left(p, p^{\prime}, q ; \phi\right) U_{s}(p, x) e_{l, \mu}^{*}(q),
$$

where

$$
\begin{aligned}
& -i e \Gamma^{\mu}\left(p, p^{\prime}, q ; \phi\right)=e^{3} \int \frac{d^{4} k}{(2 \pi)^{4}} \int_{0}^{\infty} d s \int_{0}^{\infty} d u \frac{e^{-i m^{2}(s+u)}}{k^{2}-\kappa^{2}+i 0} e^{2 i k_{+}\left[s\left(p_{-}^{\prime}+k_{-}\right)+u\left(p_{-}+k_{-}\right)\right]} e^{i\left\{p_{+}^{\prime}\left(\phi_{s}-\phi\right)+\int_{\phi}^{\phi_{s}} d \phi^{\prime}\left[-\frac{e p_{\perp}^{\prime}: A_{\perp}\left(\phi^{\prime}\right)}{p_{-}^{\prime}}+\frac{e^{2} A_{\perp}^{2}\left(\phi^{\prime}\right)}{2 p_{-}^{\prime}}\right]\right\}} \\
& \times e^{-i \int_{0}^{s} d s^{\prime}\left[\boldsymbol{p}_{\perp}^{\prime}+\boldsymbol{k}_{\perp}-e \boldsymbol{A}_{\perp}\left(\boldsymbol{\phi}_{s^{\prime}}\right)\right]^{2}} e^{-i \int_{0}^{u} d u^{\prime}\left[\boldsymbol{p}_{\perp}+\boldsymbol{k}_{\perp}-e \boldsymbol{A}_{\perp}\left(\boldsymbol{\phi}_{u^{\prime}}\right)\right]^{2}} \\
& \left.\times e^{i\left\{-p_{+}\left(\phi_{u}-\phi\right)-\int_{\phi}^{\phi_{u}} d \phi^{\prime}\left[-\frac{e p_{\perp} \cdot A_{\perp}\left(\phi^{\prime}\right)}{p_{-}}+\frac{e^{2} A_{\perp}^{2}\left(\phi^{\prime}\right)}{2 p_{-}}\right]\right.}\right\} M^{\mu}(\phi, k, s, u) .
\end{aligned}
$$

Here, we have introduced the quantities

$$
\begin{aligned}
& \phi_{s}=\phi+2 s\left(p_{-}^{\prime}+k_{-}\right), \\
& \phi_{u}=\phi-2 u\left(p_{-}+k_{-}\right),
\end{aligned}
$$

and the matrix

$$
\begin{aligned}
M^{\mu}(k, s, u ; \phi)= & \left\{1-\frac{e \hat{n}\left[\hat{A}\left(\phi_{s}\right)-\hat{A}(\phi)\right]}{2 p_{-}^{\prime}}\right\}\left[2 \pi_{p^{\prime}}^{\lambda}\left(\phi_{s}\right)+i \frac{e \hat{n} \hat{A}^{\prime}\left(\phi_{s}\right)}{p_{-}^{\prime}} n^{\lambda}+\gamma^{\lambda} \hat{k}\right] \\
& \times\left\{1+\frac{e \hat{n}\left[\hat{A}\left(\phi_{s}\right)-\hat{A}(\phi)\right]}{2\left(p_{-}^{\prime}+k_{-}\right)}\right\} \gamma^{\mu}\left\{1-\frac{e \hat{n}\left[\hat{A}\left(\phi_{u}\right)-\hat{A}(\phi)\right]}{2\left(p_{-}+k_{-}\right)}\right\} \\
& \times\left[2 \pi_{p, \lambda}\left(\phi_{u}\right)+i \frac{e \hat{n} \hat{A}^{\prime}\left(\phi_{u}\right)}{p_{-}} n_{\lambda}+\hat{k} \gamma_{\lambda}\right]\left\{1+\frac{e \hat{n}\left[\hat{A}\left(\phi_{u}\right)-\hat{A}(\phi)\right]}{2 p_{-}}\right\} .
\end{aligned}
$$


Note that the integrals in $T$ and $\boldsymbol{x}_{\perp}$ can easily be taken and enforce the conservation laws $p_{-}=p_{-}^{\prime}+q_{-}$and $\boldsymbol{p}_{\perp}=\boldsymbol{p}_{\perp}^{\prime}+\boldsymbol{q}_{\perp}$, typical of problems in a plane-wave background field. As a related remark, it is clear that the quantity $\Gamma^{\mu}\left(p, p^{\prime}, q ; \phi\right)$, unlike the corresponding vacuum expression, depends also on the plane-wave phase. Finally, the definition in Eq. (21) is consistent with the idea that computing the amplitude of the vertex in a plane wave up to one loop, one can use the substitution rule $\gamma^{\mu} \rightarrow \gamma^{\mu}+\Gamma^{\mu}\left(p, p^{\prime}, q ; \phi\right)$, as for the vertex correction in vacuum.

The phase in Eq. (22) can be written in a compact form by turning the integral from $\phi$ to $\phi_{s}$ (from $\phi$ to $\phi_{u}$ ) into an integral in $s^{\prime}\left(u^{\prime}\right)$ such as that in the second line of Eq. (22). By exponentiating also the denominator $k^{2}-\kappa^{2}+i 0$ in the photon propagator, the quantity $\Gamma^{\mu}\left(p, p^{\prime}, q ; \phi\right)$ can be written as

$$
\begin{aligned}
\Gamma^{\mu}\left(p, p^{\prime}, q ; \phi\right)= & e^{2} \int \frac{d^{4} k}{(2 \pi)^{4}} \int_{0}^{\infty} d s \int_{0}^{\infty} d u \\
& \times \int_{0}^{\infty} d t e^{i S k^{2}-i \kappa^{2} t+2 i(k \tilde{F})} M^{\mu}(k, s, u ; \phi),
\end{aligned}
$$

where $S=u+s+t$ and

$$
\tilde{F}^{\mu}=\int_{0}^{s} d s^{\prime} \pi_{p^{\prime}}^{\mu}\left(\phi_{s^{\prime}}\right)+\int_{0}^{u} d u^{\prime} \pi_{p}^{\mu}\left(\phi_{u^{\prime}}\right) .
$$

As the next step, we can perform the integrals in $d^{4} k$ analytically by shifting the four-momentum $k^{\mu}$ by setting $k^{\prime \mu}=k^{\mu}+\tilde{F}^{\mu} / S$, which, since all components of $\tilde{F}^{\mu}$ except $\tilde{F}_{-}$depend on $k_{-}$, implies that $k^{\mu}=k^{\prime \mu}-\tilde{G}^{\mu} / S$, where

$$
\tilde{G}^{\mu}=\int_{0}^{s} d s^{\prime} \pi_{p^{\prime}}^{\mu}\left(\tilde{\psi}_{s^{\prime}}\right)+\int_{0}^{u} d u^{\prime} \pi_{p}^{\mu}\left(\tilde{\psi}_{u^{\prime}}\right),
$$

such that $\tilde{G}_{-}=\tilde{F}_{-}=s p_{-}^{\prime}+u p_{-}$. Here, we have introduced the two shifted phases

$$
\begin{aligned}
& \tilde{\psi}_{s}=\phi+2 s \tau_{-}^{\prime}+2 s k_{-}, \\
& \tilde{\psi}_{u}=\phi-2 u \tau_{-}-2 u k_{-},
\end{aligned}
$$

where

$$
\begin{aligned}
& \tau_{-}^{\prime}=p_{-}^{\prime}-\frac{\tilde{G}_{-}}{S}=\frac{t p_{-}^{\prime}-u q_{-}}{S}, \\
& \tau_{-}=p_{-}-\frac{\tilde{G}_{-}}{S}=\frac{t p_{-}+s q_{-}}{S} .
\end{aligned}
$$

After the shift of the four-momentum $k^{\mu}$, we can write $\Gamma^{\mu}\left(p, p^{\prime}, q ; \phi\right)$ in the form

$$
\begin{aligned}
\Gamma^{\mu}\left(p, p^{\prime}, q ; \phi\right)= & e^{2} \int_{0}^{\infty} d s d u d t \\
& \times \int \frac{d^{4} k}{(2 \pi)^{4}} e^{-i \kappa^{2} t-i \frac{\tilde{G}^{2}}{S}+i S k^{2}} \tilde{L}\left(\tilde{Q}^{\prime \lambda}+\gamma^{\lambda} \hat{k}\right) \\
& \times \tilde{C}^{\mu}\left(\tilde{Q}_{\lambda}+\hat{k} \gamma_{\lambda}\right) \tilde{R},
\end{aligned}
$$

where

$$
\begin{gathered}
\tilde{L}=1-\frac{e \hat{n}\left[\hat{A}\left(\tilde{\psi}_{s}\right)-\hat{A}(\phi)\right]}{2 p_{-}^{\prime}}, \\
\tilde{Q}^{\lambda}=2 \pi_{p}^{\lambda}\left(\tilde{\psi}_{u}\right)+i \frac{e \hat{n} \hat{A}^{\prime}\left(\tilde{\psi}_{u}\right)}{p_{-}} n^{\lambda}-\frac{\hat{\tilde{G}}}{S} \gamma^{\lambda} \\
\tilde{C}^{\mu}=\left\{1+\frac{e \hat{n}\left[\hat{A}\left(\tilde{\psi}_{s}\right)-\hat{A}(\phi)\right]}{2\left(\tau_{-}^{\prime}+k_{-}\right)}\right\} \gamma^{\mu}\left\{1-\frac{e \hat{n}\left[\hat{A}\left(\tilde{\psi}_{u}\right)-\hat{A}(\phi)\right]}{2\left(\tau_{-}+k_{-}\right)}\right\}, \\
\tilde{Q}^{\prime \lambda}=2 \pi_{p^{\prime}}^{\lambda}\left(\tilde{\psi}_{s}\right)+i \frac{e \hat{n} \hat{A}^{\prime}\left(\tilde{\psi}_{s}\right)}{p_{-}^{\prime}} n^{\lambda}-\gamma^{\lambda} \frac{\hat{\tilde{G}}}{S}, \\
\tilde{R}=1+\frac{e \hat{n}\left[\hat{A}\left(\tilde{\psi}_{u}\right)-\hat{A}(\phi)\right]}{2 p_{-}} .
\end{gathered}
$$

The integral in $d^{4} k$ in $\Gamma^{\mu}\left(p, p^{\prime}, q ; \phi\right)$ is complicated by the fact that the variable $k_{-}$is contained in the argument of the four-vector potential of the plane wave. Thus, we first take the integral in $d^{2} \boldsymbol{k}_{\perp}$, which is Gaussian,

$$
\begin{aligned}
\Gamma^{\mu}\left(p, p^{\prime}, q ; \phi\right)= & -i \alpha \int_{0}^{\infty} \frac{d s d u d t}{S} \int \frac{d k_{-} d k_{+}}{(2 \pi)^{2}} \\
& \times e^{-i \kappa^{2} t-i \frac{\tilde{G}^{2}}{S}+2 i S k_{-} k_{+}} \tilde{M}^{\mu}\left(k_{-}, k_{+}, s, u, t ; \phi\right),
\end{aligned}
$$

where

$$
\begin{aligned}
\tilde{M}^{\mu}\left(k_{-}, k_{+}, s, u, t ; \phi\right)= & \tilde{L}\left[\left(\tilde{Q}^{\prime \lambda}+k_{-} \gamma^{\lambda} \hat{\tilde{n}}\right) \tilde{C}^{\mu}\left(\tilde{Q}_{\lambda}+k_{-} \hat{\tilde{n}} \gamma_{\lambda}\right)\right. \\
& \left.-\frac{i}{2 S} \gamma^{\lambda} \gamma_{\perp, i} \tilde{C}^{\mu} \gamma_{\perp, i} \gamma_{\lambda}\right] \tilde{R} \\
& +k_{+} \tilde{L}\left[\gamma^{\lambda} \hat{n} \tilde{C}^{\mu}\left(\tilde{Q}_{\lambda}+k_{-} \hat{\tilde{n}} \gamma_{\lambda}\right)\right. \\
& \left.+\left(\tilde{Q}^{\prime \lambda}+k_{-} \gamma^{\lambda} \hat{\tilde{n}}\right) \tilde{C}^{\mu} \hat{n} \gamma_{\lambda}\right] \tilde{R} \\
& +k_{+}^{2} \tilde{L} \gamma^{\lambda} \hat{n} \gamma^{\mu} \hat{n} \gamma_{\lambda} \tilde{R} .
\end{aligned}
$$

Finally, the integral in $d k_{+}$results in a delta function and its first and second derivatives all evaluated at $2 S k_{-}$. This allows one then to also take the integral in $d k_{-}$and, after straightforward manipulations, the resulting expression of $\Gamma^{\mu}\left(p, p^{\prime}, q ; \phi\right)$ can be written as 


$$
\begin{aligned}
\Gamma^{\mu}\left(p, p^{\prime}, q ; \phi\right)= & -\frac{i \alpha}{4 \pi} \int_{0}^{\infty} \frac{d s d u d t}{S^{3}} e^{-i \kappa^{2} t}\left\{e^{-i \frac{\tilde{G}^{2}}{S}} \tilde{L}\left(S \tilde{Q}^{\prime \lambda} \tilde{C}^{\mu} \tilde{Q}_{\lambda}+2 i \tilde{C}^{\mu}\right) \tilde{R}\right. \\
& \left.+\frac{i}{2} \frac{d}{d k_{-}}\left[e^{-i \frac{\tilde{G}^{2}}{S}} \tilde{L}\left(\gamma^{\lambda} \hat{n} \tilde{C}^{\mu} \tilde{Q}_{\lambda}+\tilde{Q}^{\prime \lambda} \tilde{C}^{\mu} \hat{n} \gamma_{\lambda}\right) \tilde{R}\right]+\frac{\hat{n}}{S} n^{\mu} \frac{d^{2}}{d k_{-}^{2}}\left(e^{-i \frac{\tilde{G}^{2}}{S}}\right)\right\}\left.\right|_{k_{-}=0} \\
= & -\frac{i \alpha}{4 \pi} \int_{0}^{\infty} \frac{d s d u d t}{S^{3}} e^{-i \kappa^{2} t-i \tilde{G}^{2}} \\
& \times\left\{\tilde{L}\left[S \tilde{Q}^{\prime \lambda} \tilde{C}^{\mu} \tilde{Q}_{\lambda}+2 i \tilde{C}^{\mu}+\frac{1}{2 S} \frac{d \tilde{G}^{2}}{d k_{-}}\left(\gamma^{\lambda} \hat{n} \tilde{C}^{\mu} \tilde{Q}_{\lambda}+\tilde{Q}^{\prime \lambda} \tilde{C}^{\mu} \hat{n} \gamma_{\lambda}\right)\right] \tilde{R}\right. \\
& \left.+\frac{i}{2} \frac{d}{d k_{-}}\left[\tilde{L}\left(\gamma^{\lambda} \hat{n} \tilde{C}^{\mu} \tilde{Q}_{\lambda}+\tilde{Q}^{\prime \lambda} \tilde{C}^{\mu} \hat{n} \gamma_{\lambda}\right) \tilde{R}\right]-\frac{\hat{n}}{S^{2}} n^{\mu}\left[\frac{1}{S}\left(\frac{d \tilde{G}^{2}}{d k_{-}}\right)^{2}+i \frac{\left.d^{2} \tilde{G}^{2}\right]}{d k_{-}^{2}}\right]\right\}\left.\right|_{k_{-}=0}
\end{aligned}
$$

This expression can be further manipulated especially to simplify its matrix structure. However, it is first convenient to make the following considerations related to the Ward identity to be fulfilled by $\Gamma^{\mu}\left(p, p^{\prime}, q ; \phi\right)$ [87]. From now on we assume that $q_{-}>0$. Thus, by using the three four-vectors

$$
\begin{gathered}
N^{\mu}=q^{\mu}-\frac{q^{2} n^{\mu}}{2 q_{-}} \\
\Lambda_{i}^{\mu}=a_{i}^{\mu}+\frac{q_{\perp, i} n^{\mu}}{q_{-}}
\end{gathered}
$$

with $i=1,2$ together with $n^{\mu}$, one can build a light-cone basis such that

$$
\eta^{\mu \nu}=\frac{N^{\mu} n^{\nu}+n^{\mu} N^{\nu}}{q_{-}}-\Lambda_{1}^{\mu} \Lambda_{1}^{\nu}-\Lambda_{2}^{\mu} \Lambda_{2}^{\nu} .
$$

Then, the quantity $\Gamma^{\mu}\left(p, p^{\prime}, q ; \phi\right) e_{l, \mu}^{*}(q)$, which is the one finally required here, can be written as [recall that we work in the Lorenz gauge where $\left(q e_{l}^{*}(q)\right)=0$ ]

$$
\begin{aligned}
\Gamma^{\mu}\left(p, p^{\prime}, q ; \phi\right) e_{l, \mu}^{*}(q)= & \frac{e_{l,-}^{*}(q)}{q_{-}} \Gamma_{q}\left(p, p^{\prime}, q ; \phi\right)-\frac{q^{2} e_{l,-}^{*}(q)}{q_{-}^{2}} \Gamma_{-}\left(p, p^{\prime}, q ; \phi\right) \\
& -\left(\Gamma\left(p, p^{\prime}, q ; \phi\right) \Lambda_{1}\right)\left(\Lambda_{1} e_{l}^{*}(q)\right)-\left(\Gamma\left(p, p^{\prime}, q ; \phi\right) \Lambda_{2}\right)\left(\Lambda_{2} e_{l}^{*}(q)\right) \\
= & \frac{e_{l,-}^{*}(q)}{q_{-}} \Gamma_{q}\left(p, p^{\prime}, q ; \phi\right)-\left[\frac{q^{2} e_{l,-}^{*}(q)}{q_{-}^{2}}+\frac{q_{\perp, 1}}{q_{-}}\left(\Lambda_{1} e_{l}^{*}(q)\right)+\frac{q_{\perp, 2}}{q_{-}}\left(\Lambda_{2} e_{l}^{*}(q)\right)\right] \Gamma_{-}\left(p, p^{\prime}, q ; \phi\right) \\
& +\Gamma_{\perp, 1}\left(p, p^{\prime}, q ; \phi\right)\left(\Lambda_{1} e_{l}^{*}(q)\right)+\Gamma_{\perp, 2}\left(p, p^{\prime}, q ; \phi\right)\left(\Lambda_{2} e_{l}^{*}(q)\right),
\end{aligned}
$$

where $\Gamma_{q}\left(p, p^{\prime}, q ; \phi\right)=\left(\Gamma^{\mu}\left(p, p^{\prime}, q ; \phi\right) q_{\mu}\right)$ and all other symbols are defined in analogy to the definitions given in the Introduction. Now, since the structure of the function $\Gamma^{\mu}\left(p, p^{\prime}, q ; \phi\right)$ is complicated because of the presence of the plane wave, it is clear that the components $\Gamma_{-}\left(p, p^{\prime}, q ; \phi\right)=\left(\Gamma^{\mu}\left(p, p^{\prime}, q ; \phi\right) n_{\mu}\right)$ and $\Gamma_{\perp, j}\left(p, p^{\prime}, q ; \phi\right)=$ $-\left(\Gamma^{\mu}\left(p, p^{\prime}, q ; \phi\right) a_{j, \mu}\right)$ are relatively easy to work out because the quantities $n^{\mu}$ and $a_{i}^{\mu}$ characterize the plane wave. For example, we observe that all the terms proportional to $n^{\mu}$ in $\Gamma^{\mu}\left(p, p^{\prime}, q ; \phi\right)$ can be ignored in the computation of the components $\Gamma_{-}\left(p, p^{\prime}, q ; \phi\right)$ and $\Gamma_{\perp, j}\left(p, p^{\prime}, q ; \phi\right)$.

The apparently most complicated term is, therefore, $\Gamma_{q}\left(p, p^{\prime}, q ; \phi\right)$, which is related to the fact that by itself the vertex-correction function is not gauge invariant. However, the gauge invariance of QED guarantees that the component $\Gamma_{q}\left(p, p^{\prime}, q ; \phi\right)$ of the vertex-correction function does not contribute to any transition amplitude involving on-shell external electrons/positrons. We explicitly prove this statement in the case under consideration with an incoming and an outgoing electron, the other possible cases being proved in an analogous way. First, we start back from Eq. (14) and we apply the same procedure to prove the Ward identity $[11,78,79]$. From the second equality in Eq. (14) and from the definition of $\Gamma^{\mu}\left(p, p^{\prime}, q ; \phi\right)$ in Eq. (21), we obtain

$$
\begin{aligned}
\Gamma_{q}\left(p, p^{\prime}, q ; \phi\right)= & -i e^{2} \int \frac{d^{4} k}{(2 \pi)^{4}} \frac{1}{k^{2}-\kappa^{2}+i 0} \gamma^{\lambda} \\
& \times \frac{1}{\hat{\Pi}(\phi)+\hat{k}-\hat{q}-m+i 0} \\
& \times \hat{q} \frac{1}{\hat{\Pi}(\phi)+\hat{k}-m+i 0} \gamma_{\lambda} .
\end{aligned}
$$




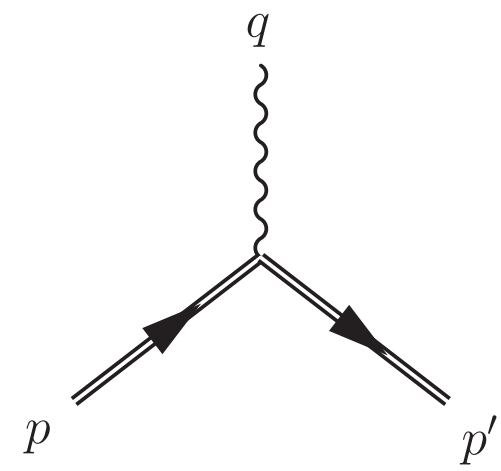

FIG. 4. The leading-order Feynman diagram corresponding to nonlinear Compton scattering of an off-shell photon. The double lines represent exact electron states in a plane wave (Volkov states) [3].

Now, by writing $\hat{q}=\hat{\Pi}(\phi)+\hat{k}-m-[\hat{\Pi}(\phi)+\hat{k}-\hat{q}-m]$ it is clear that we can express $\Gamma_{q}\left(p, p^{\prime}, q ; \phi\right)$ as the difference of two terms containing only one propagator in the plane wave:

$$
\begin{aligned}
\Gamma_{q}\left(p, p^{\prime}, q ; \phi\right)= & -i e^{2} \int \frac{d^{4} k}{(2 \pi)^{4}} \frac{1}{k^{2}-\kappa^{2}+i 0} \gamma^{\lambda} \\
& \times\left[\frac{1}{\hat{\Pi}(\phi)+\hat{k}-\hat{q}-m+i 0}\right. \\
& \left.-\frac{1}{\hat{\Pi}(\phi)+\hat{k}-m+i 0}\right] \gamma_{\lambda} .
\end{aligned}
$$

At this point, we observe that in computing, for example, the one-loop radiative corrections to nonlinear Compton scattering (see the leading-order diagram in Fig. 4, for the kinematical situation corresponding to the case under study), we also have to include the remaining diagrams listed in Fig. 5. If we indicate as $i \mathcal{M}_{s, s^{\prime}, \mu}^{(1)}\left(p, p^{\prime}, q\right) e_{l}^{* \mu}(q)$ the amplitude of the one-loop radiative corrections to nonlinear Compton scattering represented by the diagrams in Figs. 3 and 5, the gauge invariance of QED implies that $\mathcal{M}_{s, s^{\prime}, \mu}^{(1)}\left(p, p^{\prime}, q\right) q^{\mu}=0$ [91]. Since the contribution corresponding to Fig. 5(c) is by itself gauge invariant $[67,85]$, by summing the contributions from Fig. 3 and from Figs. 5(a) and 5(b), we obtain [see also Eq. (47)]

$$
\begin{aligned}
i \mathcal{M}_{s, s^{\prime}, \mu}^{(1)}\left(p, p^{\prime}, q\right) q^{\mu}= & -e^{3} \int d^{4} x \int \frac{d^{4} k}{(2 \pi)^{4}} \frac{1}{k^{2}-\kappa^{2}+i 0} \\
& \times \bar{U}_{s^{\prime}}\left(p^{\prime}, x\right)\left\{\gamma^{\lambda}\left[\frac{1}{\hat{\Pi}(\phi)+\hat{k}-m+i 0} e^{i(q x)}-e^{i(q x)} \frac{1}{\hat{\Pi}(\phi)+\hat{k}-m+i 0}\right] \gamma_{\lambda}\right. \\
& +\hat{q} e^{i(q x)} \frac{1}{\hat{\Pi}(\phi)-m+i 0} \gamma^{\lambda} \frac{1}{\hat{\Pi}(\phi)+\hat{k}-m+i 0} \gamma_{\lambda} \\
& \left.+\gamma^{\lambda} \frac{1}{\hat{\Pi}(\phi)+\hat{k}-m+i 0} \gamma_{\lambda} \frac{1}{\hat{\Pi}(\phi)-m+i 0} \hat{q} e^{i(q x)}\right\} U_{s}(p, x) .
\end{aligned}
$$

Now, by using the fact that $[\hat{\Pi}(\phi)-m] U_{s}(p, x)=$ $[\hat{\Pi}(\phi)-m] U_{s^{\prime}}\left(p^{\prime}, x\right)=0$, we first replace $\hat{q}$ with $\hat{\Pi}(\phi)+$ $\hat{q}-m(m-\hat{\Pi}(\phi)+\hat{q})$ in the third (fourth) line of this equation, and then we move the exponential $\exp [i(q x)]$ to the left of all other operators by exploiting the identity in Eq. (1). The result is
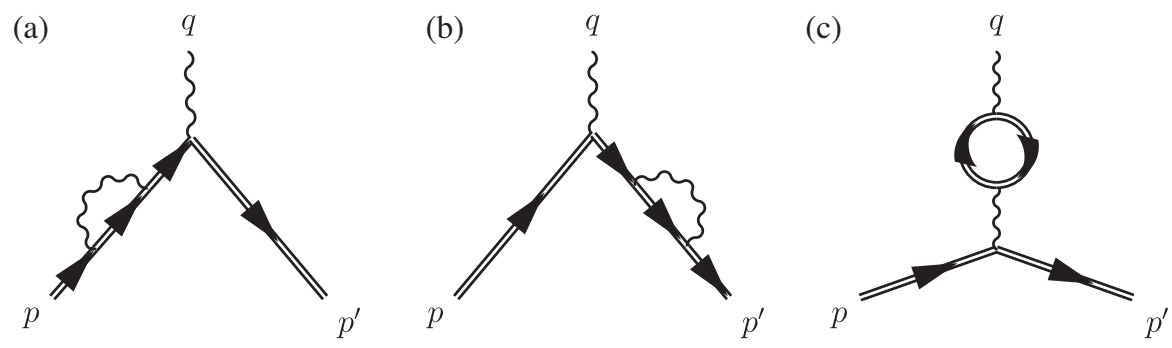

FIG. 5. The one-loop Feynman diagrams corresponding, together with the one-loop vertex correction in Fig. 3, to the leading-order radiative corrections of nonlinear Compton scattering of an off-shell photon. (a) and (b) show the contributions arising from the insertion of the mass operator in the incoming and in the outgoing electron line, respectively, whereas (c) shows the contribution arising from the insertion of the polarization operator in the photon line. The double lines represent exact electron states and propagator in a plane wave (Volkov states and propagator, respectively) [3]. 


$$
\begin{aligned}
i \mathcal{M}_{s, s^{\prime}, \mu}^{(1)}\left(p, p^{\prime}, q\right) q^{\mu}= & -e^{3} \int d^{4} x \int \frac{d^{4} k}{(2 \pi)^{4}} \frac{e^{i(q x)}}{k^{2}-\kappa^{2}+i 0} \\
& \times \bar{U}_{s^{\prime}}\left(p^{\prime}, x\right)\left\{\gamma^{\lambda}\left[\frac{1}{\hat{\Pi}(\phi)+\hat{k}-\hat{q}-m+i 0}-\frac{1}{\hat{\Pi}(\phi)+\hat{k}-m+i 0}\right] \gamma_{\lambda}\right. \\
& \left.+\gamma^{\lambda} \frac{1}{\hat{\Pi}(\phi)+\hat{k}-m+i 0} \gamma_{\lambda}-\gamma^{\lambda} \frac{1}{\hat{\Pi}(\phi)+\hat{k}-\hat{q}-m+i 0} \gamma_{\lambda}\right\} U_{s}(p, x),
\end{aligned}
$$

which indeed vanishes identically. This result indicates that gauge invariance implies that the component $\Gamma_{q}\left(p, p^{\prime}, q ; \phi\right)$ can be ignored as it will always be compensated by the corresponding contributions arising from the mass operators [some properties of $\Gamma_{q}\left(p, p^{\prime}, q ; \phi\right)$ are discussed in the Appendix].

At this point, we can consider the other component $\Gamma_{-}\left(p, p^{\prime}, q ; \phi\right)$, whose structure is particularly easy. In fact, starting from Eq. (41), we have that

$$
\begin{aligned}
\Gamma_{-}\left(p, p^{\prime}, q ; \phi\right)= & -\frac{i \alpha}{4 \pi} \int_{0}^{\infty} \frac{d s d u d t}{S^{3}} e^{-i \kappa^{2} t-i \frac{G^{2}}{S}}\left(S L Q^{\prime \lambda} \hat{n} Q_{\lambda} R+2 i \hat{n}\right) \\
= & -\frac{i \alpha}{2 \pi} \int_{0}^{\infty} \frac{d s d u d t}{S^{3}} e^{-i \kappa^{2} t-i \frac{G^{2}}{S}}\left[\left(2 S\left(\pi_{s} \pi_{u}\right)+\frac{G^{2}}{S}+i\right) \hat{n}-2 G_{-}\left(\hat{\pi}_{s} R+L \hat{\pi}_{u}\right)\right. \\
& \left.-\hat{G} \hat{\pi}_{s} \hat{n}-\hat{n} \hat{\pi}_{u} \hat{G}+2 \tau_{-} L \hat{G}+2 \tau_{-}^{\prime} \hat{G} R+2 \frac{G_{-}}{S} \hat{G}-\frac{G_{-}^{2}}{S} \frac{\hat{\Delta}_{s} \hat{n} \hat{\Delta}_{u}}{p_{-} p_{-}^{\prime}}\right],
\end{aligned}
$$

where

$$
\begin{gathered}
\pi_{s}^{\mu}=\pi_{p^{\prime}}^{\mu}\left(\psi_{s}\right), \quad \Delta_{s}^{\mu}=e\left[A^{\mu}\left(\psi_{s}\right)-A^{\mu}(\phi)\right], \quad L=1-\frac{\hat{n} \hat{\Delta}_{s}}{2 p_{-}^{\prime}}, \quad \psi_{s}=\phi+2 s \tau_{-}^{\prime}, \\
\pi_{u}^{\mu}=\pi_{p}^{\mu}\left(\psi_{u}\right), \quad \Delta_{u}^{\mu}=e\left[A^{\mu}\left(\psi_{u}\right)-A^{\mu}(\phi)\right], \quad R=1+\frac{\hat{n} \hat{\Delta}_{u}}{2 p_{-}}, \quad \psi_{u}=\phi-2 u \tau_{-}, \\
C^{\mu}=\left(1+\frac{\hat{n} \hat{\Delta}_{s}}{2 \tau_{-}^{\prime}}\right) \gamma^{\mu}\left(1-\frac{\hat{n} \hat{\Delta}_{u}}{2 \tau_{-}}\right), \quad G^{\mu}=\int_{0}^{s} d s^{\prime} \pi_{p^{\prime}}^{\mu}\left(\psi_{s^{\prime}}\right)+\int_{0}^{u} d u^{\prime} \pi_{p}^{\mu}\left(\psi_{u^{\prime}}\right), \\
Q^{\lambda}=2 \pi_{u}^{\lambda}+i \frac{e \hat{n} \hat{A}^{\prime}\left(\psi_{u}\right)}{p_{-}} n^{\lambda}-\frac{\hat{G}}{S} \gamma^{\lambda}, \quad Q^{\prime \lambda}=2 \pi_{s}^{\lambda}+i \frac{e \hat{n} \hat{A}^{\prime}\left(\psi_{s}\right)}{p_{-}^{\prime}} n^{\lambda}-\gamma^{\lambda} \frac{\hat{G}}{S} .
\end{gathered}
$$

As we have mentioned, in order to compute the components $\Gamma_{\perp, j}\left(p, p^{\prime}, q ; \phi\right)=-\left(\Gamma^{\mu}\left(p, p^{\prime}, q ; \phi\right) a_{j, \mu}\right)$ we can effectively assume that the matrix $\hat{n}$ anticommutes with $\gamma^{\mu}$. In the following four equations, with an abuse of notation, we use the equal symbol also for two matrices that are equal to each other up to terms proportional to $n^{\mu}$, which can anyway be ignored in the computation of $\Gamma_{\perp, j}\left(p, p^{\prime}, q ; \phi\right)$. Going through the terms in Eq. (41) in order of complexity, one can easily show that

$$
\begin{aligned}
& L C^{\mu} R=\gamma^{\mu}+\frac{G_{-}}{2 p_{-}^{\prime} \tau_{-}^{\prime} S} \hat{n} \hat{\Delta}_{s} \gamma^{\mu}-\frac{G_{-}}{2 p_{-} \tau_{-} S} \gamma^{\mu} \hat{n} \hat{\Delta}_{u}, \\
& L\left(\gamma^{\lambda} \hat{n} C^{\mu} Q_{\lambda}+Q^{\prime \lambda} C^{\mu} \hat{n} \gamma_{\lambda}\right) R=-\frac{2 \tau_{-}}{p_{-}^{\prime}} \hat{n} \hat{\Delta}_{s} \gamma^{\mu}+\frac{2 \tau_{-}^{\prime}}{p_{-}} \gamma^{\mu} \hat{n} \hat{\Delta}_{u}-\frac{4 G_{-}}{S} \gamma^{\mu}+\frac{4 G^{\mu}}{S} \hat{n} \\
& -2 \hat{n} \gamma^{\mu} \hat{\pi}_{s}-2 \hat{\pi}_{u} \gamma^{\mu} \hat{n}, \\
& \frac{d}{d k_{-}}\left[\tilde{L}\left(\gamma^{\lambda} \hat{n} \tilde{C}^{\mu} \tilde{Q}_{\lambda}+\tilde{Q}^{\prime \lambda} \tilde{C}^{\mu} \hat{n} \gamma_{\lambda}\right) \tilde{R}\right]_{k_{-}=0}=8\left(\frac{G_{1}^{\mu}}{S}-\frac{s \tau_{-}}{p_{-}^{\prime}} \mathcal{A}_{s}^{\prime \mu}+\frac{u \tau_{-}^{\prime}}{p_{-}} \mathcal{A}_{u}^{\prime \mu}\right) \hat{n} \\
& +4 s\left(1+\frac{\tau_{-}}{p_{-}^{\prime}}\right) \hat{n} \gamma^{\mu} \hat{\mathcal{A}}_{s}^{\prime}-4 u\left(1+\frac{\tau_{-}^{\prime}}{p_{-}}\right) \hat{\mathcal{A}}_{u}^{\prime} \gamma^{\mu} \hat{n},
\end{aligned}
$$




$$
\begin{aligned}
L Q^{\prime \lambda} C^{\mu} Q_{\lambda} R= & 4\left(\pi_{s} \pi_{u}\right)\left(\gamma^{\mu}+\frac{G_{-}}{2 p_{-}^{\prime} \tau_{-}^{\prime} S} \hat{n} \hat{\Delta}_{s} \gamma^{\mu}-\frac{G_{-}}{2 p_{-} \tau_{-} S} \gamma^{\mu} \hat{n} \hat{\Delta}_{u}\right) \\
& +2 i \frac{\tau_{-}}{p_{-}^{\prime}} \hat{n} \hat{\mathcal{A}}_{s}^{\prime} \gamma^{\mu}+2 i \frac{\tau_{-}^{\prime}}{p_{-}} \gamma^{\mu} \hat{n} \hat{\mathcal{A}}_{u}^{\prime}-\frac{2}{S} L C^{\mu} \hat{G} \hat{\pi}_{s} R-\frac{2}{S} L \hat{\pi}_{u} \hat{G} C^{\mu} R \\
& -\frac{2}{S^{2}} L \hat{G}\left(\gamma^{\mu}+\frac{\gamma^{\mu} \hat{\Delta}_{s} \hat{n}}{2 \tau_{-}^{\prime}}-\frac{\hat{\Delta}_{u} \hat{n} \gamma^{\mu}}{2 \tau_{-}}\right) \hat{G} R,
\end{aligned}
$$

where

$$
\begin{aligned}
G_{1}^{\mu} & =\frac{d}{d \phi}\left[\int_{0}^{s} d s^{\prime} s^{\prime} \pi_{p^{\prime}}^{\mu}\left(\psi_{s^{\prime}}\right)-\int_{0}^{u} d u^{\prime} u^{\prime} \pi_{p}^{\mu}\left(\psi_{u^{\prime}}\right)\right] \\
& =\frac{1}{2 \tau_{-}^{\prime}}\left[s \pi_{p^{\prime}}^{\mu}\left(\psi_{s}\right)-\int_{0}^{s} d s^{\prime} \pi_{p^{\prime}}^{\mu}\left(\psi_{s^{\prime}}\right)\right]+\frac{1}{2 \tau_{-}}\left[u \pi_{p}^{\mu}\left(\psi_{u}\right)-\int_{0}^{u} d u^{\prime} \pi_{p}^{\mu}\left(\psi_{u^{\prime}}\right)\right]
\end{aligned}
$$

and

$$
\mathcal{A}_{s / u}^{\mu}=e A^{\mu}\left(\psi_{s / u}\right)
$$

(the prime on these quantities indicates the derivative with respect to $\phi$ ).

In this way, we obtain the following expressions of the transverse components $\Gamma_{\perp, i}\left(p, p^{\prime}, q ; \phi\right)$ :

$$
\begin{aligned}
\Gamma_{\perp, j}\left(p, p^{\prime}, q ; \phi\right)= & \frac{i \alpha}{2 \pi} \int_{0}^{\infty} \frac{d s d u d t}{S^{3}} e^{-i \kappa^{2} t-i G^{2}} \\
& \times\left\{\left(2 S\left(\pi_{s} \pi_{u}\right)+i\right)\left(\hat{a}_{j}+\frac{G_{-}}{2 p_{-}^{\prime} \tau_{-}^{\prime} S} \hat{n} \hat{\Delta}_{s} \hat{a}_{j}-\frac{G_{-}}{2 p_{-} \tau_{-} S} \hat{a}_{j} \hat{n} \hat{\Delta}_{u}\right)\right. \\
& -L\left(C a_{j}\right) \hat{G} \hat{\pi}_{s} R-L \hat{\pi}_{u} \hat{G}\left(C a_{j}\right) R-\frac{1}{S} L \hat{G}\left(\hat{a}_{j}+\frac{\hat{a}_{j} \hat{\Delta}_{s} \hat{n}}{2 \tau_{-}^{\prime}}-\frac{\hat{\Delta}_{u} \hat{n} \hat{a}_{j}}{2 \tau_{-}}\right) \hat{G} R \\
& -\frac{2\left(G G_{1}\right)}{S}\left(\frac{\tau_{-}}{p_{-}^{\prime}} \hat{n} \hat{\Delta}_{s} \hat{a}_{j}-\frac{\tau_{-}^{\prime}}{p_{-}} \hat{a}_{j} \hat{n} \hat{\Delta}_{u}+\frac{2 G_{-}}{S} \hat{a}_{j}-\frac{2\left(G a_{j}\right)}{S} \hat{n}+\hat{n} \hat{a}_{j} \hat{\pi}_{s}+\hat{\pi}_{u} \hat{a}_{j} \hat{n}\right) \\
& +2 i\left(\frac{\left(G_{1} a_{j}\right)}{S}+s\left(\mathcal{A}_{s}^{\prime} a_{j}\right)-u\left(\mathcal{A}_{u}^{\prime} a_{j}\right)\right) \hat{n} \\
& \left.+i\left[s-(u+t) \frac{\left.\tau_{-}\right]}{p_{-}^{\prime}}\right] \hat{\mathcal{A}}_{s}^{\prime} \hat{n} \hat{a}_{j}-i\left[u-(s+t) \frac{\left.\tau_{-}^{\prime}\right]}{p_{-}}\right] \hat{a}_{j} \hat{n} \hat{\mathcal{A}}_{u}^{\prime}\right\} .
\end{aligned}
$$

Equations (45), (50), and (61) are the main results of the paper and, as it can easily be shown, they reduce to the result in vacuum as, e.g., on page 339 of Ref. [87] [as it is shown in the Appendix, the component $\Gamma_{q}\left(p, p^{\prime}, q ; \phi\right)$ vanishes in vacuum]. We notice that the preexponential matrices in all terms feature symmetry properties such that they can all be written as the sum of two classes of terms with the second one, being obtained from the first one as follows: (1) by taking the Dirac conjugate, and (2) by swapping all indexes $s$ and $u$ in each quantity. We have exploited this symmetry in the computations presented below. Finally, we observe that all the terms in Eqs. (45), (50), and (61) have at most three gamma matrices except the three terms on the third line of Eq. (61) [92]. The three terms in the third line of Eq. (61) can easily be reduced to expressions containing at most five gamma matrices:

$$
\begin{aligned}
L\left(C a_{j}\right) \hat{G} \hat{\pi}_{s} R= & \left(\hat{a}_{j}+\frac{G_{-}}{2 S p_{-}^{\prime} \tau_{-}^{\prime}} \hat{n} \hat{\Delta}_{s} \hat{a}_{j}-\frac{G_{-}}{2 S p_{-} \tau_{-}} \hat{a}_{j} \hat{n} \hat{\Delta}_{u}\right) \hat{G} \hat{\pi}_{s} \\
& +\left(\hat{a}_{j}+\frac{G_{-}}{2 S p_{-}^{\prime} \tau_{-}^{\prime}} \hat{n} \hat{\Delta}_{s} \hat{a}_{j}\right)\left(p_{-}^{\prime} \hat{G}-G_{-} \hat{\pi}_{s}\right) \frac{\hat{\Delta}_{u}}{p_{-}} \\
& +\frac{\hat{a}_{j} \hat{n}}{2 p_{-} \tau_{-}}\left[2\left(G_{-}\left(\Delta_{u} \pi_{s}\right)-p_{-}^{\prime}\left(\Delta_{u} G\right)\right) \hat{\Delta}_{u}-\left(G_{-} \Delta_{u}^{2}+2 \tau_{-}\left(\Delta_{u} G\right)\right) \hat{\pi}_{s}+\left(p_{-}^{\prime} \Delta_{u}^{2}+2 \tau_{-}\left(\Delta_{u} \pi_{s}\right)\right) \hat{G}\right],
\end{aligned}
$$




$$
\begin{aligned}
L \hat{\pi}_{u} \hat{G}\left(C a_{j}\right) R= & \hat{\pi}_{u} \hat{G}\left(\hat{a}_{j}+\frac{G_{-}}{2 S p_{-}^{\prime} \tau_{-}^{\prime}} \hat{n} \hat{\Delta}_{s} \hat{a}_{j}-\frac{G_{-}}{2 S p_{-} \tau_{-}} \hat{a}_{j} \hat{n} \hat{\Delta}_{u}\right) \\
+ & \frac{\hat{\Delta}_{s}}{p_{-}^{\prime}}\left(p_{-} \hat{G}-G_{-} \hat{\pi}_{u}\right)\left(\hat{a}_{j}-\frac{G_{-}}{2 S p_{-} \tau_{-}} \hat{a}_{j} \hat{n} \hat{\Delta}_{u}\right) \\
+ & {\left[2\left(G_{-}\left(\Delta_{s} \pi_{u}\right)-p_{-}\left(\Delta_{s} G\right)\right) \hat{\Delta}_{s}-\left(G_{-} \Delta_{s}^{2}+2 \tau_{-}^{\prime}\left(\Delta_{s} G\right)\right) \hat{\pi}_{u}+\left(p_{-} \Delta_{s}^{2}+2 \tau_{-}^{\prime}\left(\Delta_{s} \pi_{u}\right)\right) \hat{G}\right] \frac{\hat{n} \hat{a}_{j}}{2 p_{-}^{\prime} \tau_{-}^{\prime}}, \quad(63) } \\
L \hat{G}\left(\hat{a}_{j}+\frac{\hat{a}_{j} \hat{\Delta_{s}} \hat{n}}{2 \tau_{-}^{\prime}}-\frac{\hat{\Delta}_{u} \hat{n} \hat{a}_{j}}{2 \tau_{-}}\right) \hat{G} R= & \frac{G_{-}}{2}\left(\frac{\hat{G} \hat{a}_{j} \hat{\Delta}_{s} \hat{n} \hat{\Delta}_{u}}{p_{-} \tau_{-}^{\prime}}+\frac{\hat{\Delta}_{s} \hat{n} \hat{\Delta}_{u} \hat{a}_{j} \hat{G}}{p_{-}^{\prime} \tau_{-}}\right)+G_{-}\left(\frac{\hat{a}_{j} \hat{\Delta_{s}} \hat{G}}{\tau_{-}^{\prime}}+\frac{\hat{G} \hat{\Delta}_{u} \hat{a}_{j}}{\tau_{-}}\right) \\
& +\left[\left(p_{-}^{\prime}+\tau_{-}^{\prime}\right)\left(G a_{j}\right)+G_{-}\left(\Delta_{s} a_{j}\right)\right] \frac{\hat{\Delta}_{s} \hat{n} \hat{G}}{p_{-}^{\prime} \tau_{-}^{\prime}}+\left[\left(p_{-}+\tau_{-}\right)\left(G a_{j}\right)+G_{-}\left(\Delta_{u} a_{j}\right)\right] \frac{\hat{G} \hat{n} \hat{\Delta_{u}}}{p_{-} \tau_{-}}-G^{2} \hat{a}_{j} \\
& -\left[\left(p_{-}^{\prime}+\tau_{-}^{\prime}\right) G^{2}+\frac{\tau_{-}^{\prime} G_{-}^{2}}{p_{-} \tau_{-}} \Delta_{u}^{2}\right] \frac{\hat{\Delta}_{s} \hat{n} \hat{a}_{j}}{2 p_{-}^{\prime} \tau_{-}^{\prime}}\left[\left(p_{-}+\tau_{-}\right) G^{2}+\frac{\tau_{-} G_{-}^{2}}{p_{-}^{\prime} \tau_{-}^{\prime}} \Delta_{s}^{2}\right] \frac{\hat{a}_{j} \hat{n} \hat{\Delta_{u}}}{2 p_{-} \tau_{-}}+2\left(G a_{j}\right) \hat{G} \\
& -\left[G_{-} \Delta_{s}^{2}+2 p_{-}^{\prime}\left(G \Delta_{s}\right)\right] \frac{\hat{a}_{j} \hat{n} \hat{G}}{2 p_{-}^{\prime} \tau_{-}^{\prime}}-\left[G_{-} \Delta_{u}^{2}+2 p_{-}\left(G \Delta_{u}\right)\right] \frac{\hat{G} \hat{n} \hat{a}_{j}}{2 p_{-} \tau_{-}} \\
& +\frac{G_{-}}{p_{-} p_{-}^{\prime}}\left[\left(G a_{j}\right)+\frac{G_{-}}{\tau_{-}^{\prime}}\left(\Delta_{s} a_{j}\right)+\frac{G_{-}}{\tau_{-}}\left(\Delta_{u} a_{j}\right)\right] \hat{\Delta}_{s} \hat{n} \hat{\Delta}_{u}-G^{2}\left[\frac{\left(\Delta_{s} a_{j}\right)}{\tau_{-}^{\prime}}+\frac{\left(\Delta_{u} a_{j}\right)}{\tau_{-}}\right] \hat{n} .
\end{aligned}
$$

Below, we will further investigate the structure of the vertex correction and discuss its divergences.

\section{GAUGE-INVARIANCE PROPERTIES OF THE VERTEX-CORRECTION FUNCTION}

The first aspect we would like to discuss is the gauge invariance of the expression of $\Gamma_{s, s^{\prime}, l}\left(p, p^{\prime}, q\right)$ obtained above. On the one hand, it is clear that $\Gamma_{s, s^{\prime}, l}\left(p, p^{\prime}, q\right)$ is invariant under a gauge transformation of the plane wave four-vector potential, as it can be proved by replacing $A^{\mu}(\phi)$ with $A^{\mu}(\phi)+\partial^{\mu} f(\phi)=A^{\mu}(\phi)+n^{\mu} f^{\prime}(\phi)$, with $f(\phi)$ being an arbitrary function of $\phi$ [we recall, in particular, that $\pi_{p}^{\mu}(\phi)$ is the kinetic four-momentum of an electron in a plane wave and it is therefore gauge invariant; see Eq. (18)]. Now, concerning a gauge transformation of the radiation field and, in particular, of the external photon, we have already discussed that $\Gamma^{\mu}\left(p, p^{\prime}, q ; \phi\right)$ is written in a form that automatically fulfills the Ward identity, in such a way that one-loop radiative corrections are gauge invariant. In addition, we study here the effect of the additional term $\delta \Gamma_{s, s^{\prime}, l}^{(\xi)}\left(p, p^{\prime}, q\right)$ brought about by considering the photon propagator $D^{(\xi) \lambda \nu}(x)$ [87],

$$
D^{(\xi) \lambda \nu}(x)=\int \frac{d^{4} k}{(2 \pi)^{4}} \frac{e^{-i(k x)}}{k^{2}-\kappa^{2}+i 0}\left[\eta^{\lambda \nu}+\left(1-\frac{1}{\xi}\right) \frac{k^{\lambda} k^{\nu}}{k^{2}-\kappa^{2}+i 0}\right],
$$

in an arbitrary gauge parametrized by the constant $\xi$ (the Lorenz gauge corresponds to $\xi=1$ ). It is clear from Eq. (14) that

$$
\begin{aligned}
\delta \Gamma_{s, s^{\prime}, l}^{(\xi)}\left(p, p^{\prime}, q\right)= & -i e^{2}\left(1-\frac{1}{\xi}\right) \int d^{4} x \int \frac{d^{4} k}{(2 \pi)^{4}} \frac{1}{\left(k^{2}-\kappa^{2}+i 0\right)^{2}} \\
& \times \bar{U}_{s^{\prime}}\left(p^{\prime}, x\right) \hat{k} \frac{1}{\hat{\Pi}(\phi)+\hat{k}-m+i 0} e^{i(q x)} \hat{e}_{l}^{*}(q) \frac{1}{\hat{\Pi}(\phi)+\hat{k}-m+i 0} \hat{k} U_{s}(p, x) .
\end{aligned}
$$

Now, since $[\hat{\Pi}(\phi)-m] U_{s}(p, x)=[\hat{\Pi}(\phi)-m] U_{s^{\prime}}\left(p^{\prime}, x\right)=0$, we have that $\bar{U}_{s^{\prime}}\left(p^{\prime}, x\right) \hat{k}=\bar{U}_{s^{\prime}}\left(p^{\prime}, x\right)[\hat{\Pi}(\phi)+\hat{k}-m]$ and analogously $\hat{k} U_{s}(p, x)=[\hat{\Pi}(\phi)+\hat{k}-m] U_{s}(p, x)$. Thus, the two electron propagators in $\delta \Gamma_{s, s^{\prime}, l}^{(\xi)}\left(p, p^{\prime}, q\right)$ simplify, and this quantity can be written in the form

$$
\delta \Gamma_{s, s^{\prime}, l}^{(\xi)}\left(p, p^{\prime}, q\right)=Z^{(\xi)} \int d^{4} x e^{i(q x)} \bar{U}_{s^{\prime}}\left(p^{\prime}, x\right) \hat{e}_{l}^{*}(q) U_{s}(p, x)
$$


with

$$
Z^{(\xi)}=-i e^{2}\left(1-\frac{1}{\xi}\right) \int \frac{d^{4} k}{(2 \pi)^{4}} \frac{1}{\left(k^{2}-\kappa^{2}+i 0\right)^{2}}
$$

being a logarithmically divergent, gauge-dependent constant. However, since $\delta \Gamma_{s, s^{\prime}, l}^{(\xi)}\left(p, p^{\prime}, q\right)$ has exactly the same structure of the tree-level matrix element of (virtual) nonlinear Compton scattering, the constant $Z^{(\xi)}$ can be absorbed in the renormalization of the electric charge exactly as in vacuum [87]. Thus, we conclude that the gaugedependent part of the vertex correction can be absorbed in the renormalization of the electric charge, and below we will continue to work in the Lorenz gauge.

\section{CONVERGENCE PROPERTIES OF THE VERTEX-CORRECTION FUNCTION}

Analogously as the corresponding quantity in vacuum, the quantity $\Gamma^{\mu}\left(p, p^{\prime}, q ; \phi\right)$ is logarithmically divergent in the ultraviolet, as it can be ascertained from the integral in $d^{4} k$ in Eq. (14) (see, e.g., the book from [87] for the analysis of the vacuum case). Now, if we imagine expanding the exact Volkov propagators in powers of the external field (see also Fig. 3), it is clear that, since the divergence of the corresponding vacuum amplitude is logarithmic, all resulting terms depending on the field are ultraviolet convergent because the loop contains at least three vacuum electron propagators apart from the photon propagator. It is important to stress here that this does not imply that the whole field-dependent part of $\Gamma_{s, s^{\prime}, l}\left(p, p^{\prime}, q\right)$ is ultraviolet convergent because the terms dependent on the field exclusively through the external electron states are still logarithmically divergent. For this reason, the correct way to regularize the vertex correction in the plane wave is to regularize the quantity $\Gamma^{\mu}\left(p, p^{\prime}, q ; \phi\right)$ (see also Refs. [78,79]). Since the divergence at hand is only logarithmic, one first writes $\Gamma^{\mu}\left(p, p^{\prime}, q ; \phi\right)=\Gamma^{\mu}\left(p, p^{\prime}, q ; \phi\right)-$ $\Gamma_{0}^{\mu}\left(p, p^{\prime}, q\right)+\Gamma_{0}^{\mu}\left(p, p^{\prime}, q\right), \quad$ where $\quad \Gamma_{0}^{\mu}\left(p, p^{\prime}, q\right)=$ $\left.\Gamma^{\mu}\left(p, p^{\prime}, q ; \phi\right)\right|_{A^{\mu}(\phi)=0}$, and notices that $\Gamma^{\mu}\left(p, p^{\prime}, q ; \phi\right)-$ $\Gamma_{0}^{\mu}\left(p, p^{\prime}, q\right)$ is ultraviolet convergent. Then, one can regularize the vacuum expression $\Gamma_{0}^{\mu}\left(p, p^{\prime}, q\right)$ exactly as in the vacuum, i.e., by subtracting the same expression evaluated for $q^{\mu}=0$ and for $\hat{p}=\hat{p}^{\prime}=m$ [87] (notice that the conservation laws in a plane wave already imply that $p^{\prime \mu}=$ $p^{\mu}$ because these four-momenta are on-shell). In conclusion, by assuming that $e$ indicates the physical electron charge, we continue by investigating the regularized vertex function $\Gamma_{R}^{\mu}\left(p, p^{\prime}, q ; \phi\right)=\Gamma^{\mu}\left(p, p^{\prime}, q ; \phi\right)-\left.\Gamma_{0}^{\mu}(p, p, 0)\right|_{\hat{p}=m}$. By using the master integrals

$$
\begin{gathered}
\int \frac{d^{4} k}{(2 \pi)^{4}} e^{i S k^{2}}=-\frac{i}{16 \pi^{2} S^{2}}, \\
\int \frac{d^{4} k}{(2 \pi)^{4}} k^{\mu} k^{\nu} e^{i S k^{2}}=\frac{\eta^{\mu \nu}}{4} \int \frac{d^{4} k}{(2 \pi)^{4}} k^{2} e^{i S k^{2}}=\frac{\eta^{\mu \nu}}{32 \pi^{2} S^{3}},
\end{gathered}
$$

it is straightforward to take the integral in $d^{4} k$ in $\left.\Gamma_{0}^{\mu}(p, p, 0)\right|_{\hat{p}=m}$ and to obtain the result

$$
\left.\Gamma_{0}^{\mu}(p, p, 0)\right|_{\hat{p}=m}=-i \frac{\alpha}{2 \pi} \gamma^{\mu} \int_{0}^{\infty} \frac{d s d u d t}{S^{3}} e^{-i \kappa^{2} t-i \frac{(s+u)^{2}}{S} m^{2}}\left\{m^{2}\left[2 t-\frac{(s+u)^{2}}{S}\right]+i\right\} .
$$

From the derivations, it is clear that $\left.\Gamma_{0}^{\mu}(p, p, 0)\right|_{\hat{p}=m}$ has only components $\left.\Gamma_{0,-}(p, p, 0)\right|_{\hat{p}=m}$ and $\left.\Gamma_{0, \perp, j}(p, p, 0)\right|_{\hat{p}=m}$, and then that $\Gamma_{R, q}\left(p, p^{\prime}, q ; \phi\right)=\Gamma_{q}\left(p, p^{\prime}, q ; \phi\right)$, which, as we have mentioned, can be shown to vanish for $A^{\mu}(\phi)=0$ (see the Appendix).

Now, we would like to investigate the convergence properties of the proper time integrals in $\Gamma_{R,-}\left(p, p^{\prime}, q ; \phi\right)$ and $\Gamma_{R, \perp, j}\left(p, p^{\prime}, q ; \phi\right)$. It is first convenient to use the following identity [93]:

$$
\begin{aligned}
\int_{0}^{\infty} d s \int_{0}^{\infty} d u \int_{0}^{\infty} d t & =\int_{0}^{\infty} d s \int_{0}^{\infty} d u \int_{0}^{\infty} d t \int_{0}^{\infty} d S \delta(S-s-u-t) \\
& =\int_{0}^{\infty} d S \int_{0}^{S} d s \int_{0}^{S} d u \int_{0}^{S} d t \delta(S-s-u-t) \\
& =\int_{0}^{\infty} d S S^{2} \int_{0}^{1} d x \int_{0}^{1} d y \int_{0}^{1} d z \delta(1-x-y-z),
\end{aligned}
$$

where in the last line we performed the changes of variables $s=x S, u=y S$, and $t=z S$. By setting

$$
\int_{\delta} d x d y d z=\int_{0}^{1} d x \int_{0}^{1} d y \int_{0}^{1} d z \delta(1-x-y-z)
$$

it is instructive to report the expression of $\left.\Gamma_{0}^{\mu}(p, p, 0)\right|_{\hat{p}=m}$ in terms of the new variables: 


$$
\left.\Gamma_{0}^{\mu}(p, p, 0)\right|_{\hat{p}=m}=-i \frac{\alpha}{2 \pi} \gamma^{\mu} \int_{0}^{\infty} d S \int_{\delta} d x d y d z e^{-i \kappa^{2} z S-i m^{2}(x+y)^{2} S}\left\{m^{2}\left[2 z-(x+y)^{2}\right]+\frac{i}{S}\right\}
$$

because it clearly shows that only the term whose integrand is proportional to $i$ is (logarithmically) divergent (in the limit $S \rightarrow 0$ ). This divergence is related with the ultraviolet logarithmic divergence of the vertex-correction function. Keeping in mind that $z=1-x-y$ [see Eq. (73)], another divergence for $x+y \rightarrow 0$ arises for a massless photon $\left(\kappa^{2}=0\right)$, which corresponds to the infrared divergence of the vertex-correction function. By means of the above change of variables, we obtain

$$
\begin{aligned}
\Gamma_{R,-}\left(p, p^{\prime}, q ; \phi\right)= & \frac{\alpha}{2 \pi} \hat{n} \int_{0}^{\infty} \frac{d S}{S} \int_{\delta} d x d y d z e^{-i \kappa^{2} z S}\left[e^{-i g^{2} S}-e^{-i m^{2}(x+y)^{2} S}\right] \\
& -\frac{i \alpha}{2 \pi} \int_{0}^{\infty} d S \int_{\delta} d x d y d z e^{-i \kappa^{2} z S}\left\{e ^ { - i g ^ { 2 } S } \left[\left(2\left(\pi_{S} \pi_{u}\right)+g^{2}\right) \hat{n}-2 g_{-}\left(\hat{\pi}_{s} R+L \hat{\pi}_{u}\right)-\hat{g} \hat{\pi}_{s} \hat{n}-\hat{n} \hat{\pi}_{u} \hat{g}\right.\right. \\
& \left.\left.+2 \tau_{-} L \hat{g}+2 \tau_{-}^{\prime} \hat{g} R+2 g_{-} \hat{g}-g_{-}^{2} \frac{\hat{\Delta}_{s} \hat{n} \hat{\Delta}_{u}}{p_{-} p_{-}^{\prime}}\right]-m^{2} \hat{n} e^{-i m^{2}(x+y)^{2} S}\left[2 z-(x+y)^{2}\right]\right\}
\end{aligned}
$$

and

$$
\begin{aligned}
\Gamma_{R, \perp, j}\left(p, p^{\prime}, q ; \phi\right)= & -\frac{\alpha}{2 \pi} \hat{a}_{j} \int_{0}^{\infty} \frac{d S}{S} \int_{\delta} d x d y d z e^{-i \kappa^{2} z S}\left[e^{-i g^{2} S}-e^{-i m^{2}(x+y)^{2} S}\right] \\
& +\frac{i \alpha}{2 \pi} \int_{0}^{\infty} d S \int_{\delta} d x d y d z e^{-i \kappa^{2} z S}\left\langlee ^ { - i g ^ { 2 } S } \left\{ 2\left(\pi_{s} \pi_{u}\right)\left(\hat{a}_{j}+\frac{g_{-}}{2 p_{-}^{\prime} \tau_{-}^{\prime}} \hat{n} \hat{\Delta}_{s} \hat{a}_{j}-\frac{g_{-}}{2 p \tau_{-}} \hat{a}_{j} \hat{n} \hat{\Delta}_{u}\right)\right.\right. \\
& +\frac{i}{S}\left(\frac{g_{-}}{2 p_{-}^{\prime} \tau_{-}^{\prime}} \hat{n}_{\hat{\Delta}_{s}} \hat{a}_{j}-\frac{g_{-}}{2 p_{-} \tau_{-}} \hat{a}_{j} \hat{n} \hat{\Delta}_{u}\right)-L\left(C a_{j}\right) \hat{g} \hat{\pi}_{s} R-L \hat{\pi}_{u} \hat{g}\left(C a_{j}\right) R \\
& -L \hat{g}\left(\hat{a}_{j}+\frac{\hat{a}_{j} \hat{\Delta}_{s} \hat{n}}{2 \tau_{-}^{\prime}}-\frac{\hat{\Delta}_{u} \hat{n} \hat{a}_{j}}{2 \tau_{-}}\right) \hat{g} R-2 S\left(g g_{1}\right)\left(\frac{\tau_{-}}{p_{-}^{\prime}} \hat{n} \hat{\Delta}_{s} \hat{a}_{j}-\frac{\tau_{-}^{\prime}}{p_{-}} \hat{a}_{j} \hat{n} \hat{\Delta}_{u}+2 g_{-} \hat{a}_{j}-2\left(g a_{j}\right) \hat{n}\right. \\
& \left.+\hat{n} \hat{a}_{j} \hat{\pi}_{s}+\hat{\pi}_{u} \hat{a}_{j} \hat{n}\right)+2 i\left(\left(g_{1} a_{j}\right)+x\left(\mathcal{A}_{s}^{\prime} a_{j}\right)-y\left(\mathcal{A}_{u}^{\prime} a_{j}\right)\right) \hat{n}+i\left[x-(y+z) \frac{\tau_{-}}{p_{-}^{\prime}}\right] \hat{\mathcal{A}}_{s}^{\prime} \hat{n} \hat{a}_{j} \\
& \left.\left.-i\left[y-(x+z) \frac{\tau_{-}^{\prime}}{p_{-}}\right] \hat{a}_{j} \hat{n} \hat{\mathcal{A}}_{u}^{\prime}\right\}-m^{2} \hat{a}_{j} e^{-i m^{2}(x+y)^{2} S}\left[2 z-(x+y)^{2}\right]\right\rangle
\end{aligned}
$$

where

$$
\begin{aligned}
L\left(C a_{j}\right) \hat{g} \hat{\pi}_{s} R= & \left(\hat{a}_{j}+\frac{g_{-}}{2 p_{-}^{\prime} \tau_{-}^{\prime}} \hat{n} \hat{\Delta}_{s} \hat{a}_{j}-\frac{g_{-}}{2 p_{-} \tau_{-}} \hat{a}_{j} \hat{n} \hat{\Delta}_{u}\right) \hat{g} \hat{\pi}_{s} \\
& +\left(\hat{a}_{j}+\frac{g_{-}}{2 p_{-}^{\prime} \tau_{-}^{\prime}} \hat{n} \hat{\Delta}_{s} \hat{a}_{j}\right)\left(p_{-}^{\prime} \hat{g}-g_{-} \hat{\pi}_{s}\right) \frac{\hat{\Delta}_{u}}{p_{-}} \\
& +\frac{\hat{a}_{j} \hat{n}}{2 p_{-} \tau_{-}}\left[2\left(g_{-}\left(\Delta_{u} \pi_{s}\right)-p_{-}^{\prime}\left(\Delta_{u} g\right)\right) \hat{\Delta}_{u}-\left(g_{-} \Delta_{u}^{2}+2 \tau_{-}\left(\Delta_{u} g\right)\right) \hat{\pi}_{s}+\left(p_{-}^{\prime} \Delta_{u}^{2}+2 \tau_{-}\left(\Delta_{u} \pi_{s}\right)\right) \hat{g}\right], \\
L \hat{\pi}_{u} \hat{g}\left(C a_{j}\right) R= & \hat{\pi}_{u} \hat{g}\left(\hat{a}_{j}+\frac{g_{-}}{2 p_{-}^{\prime} \tau_{-}^{\prime}} \hat{n} \hat{\Delta}_{s} \hat{a}_{j}-\frac{g_{-}}{2 p_{-} \tau_{-}} \hat{a}_{j} \hat{n} \hat{\Delta}_{u}\right) \\
& +\frac{\hat{\Delta}_{s}}{p_{-}^{\prime}}\left(p_{-} \hat{g}-g_{-} \hat{\pi}_{u}\right)\left(\hat{a}_{j}-\frac{g_{-}}{2 p_{-} \tau_{-}} \hat{a}_{j} \hat{n} \hat{\Delta}_{u}\right) \\
& +\left[2\left(g_{-}\left(\Delta_{s} \pi_{u}\right)-p_{-}\left(\Delta_{s} g\right)\right) \hat{\Delta}_{s}-\left(g_{-} \Delta_{s}^{2}+2 \tau_{-}^{\prime}\left(\Delta_{s} g\right)\right) \hat{\pi}_{u}+\left(p_{-} \Delta_{s}^{2}+2 \tau_{-}^{\prime}\left(\Delta_{s} \pi_{u}\right)\right) \hat{g}\right] \frac{\hat{n} \hat{a}_{j}}{2 p_{-}^{\prime} \tau_{-}^{\prime}},
\end{aligned}
$$




$$
\begin{aligned}
L \hat{g}\left(\hat{a}_{j}+\frac{\hat{a}_{j} \hat{\Delta}_{s} \hat{n}}{2 \tau_{-}^{\prime}}-\frac{\hat{\Delta}_{u} \hat{n} \hat{a}_{j}}{2 \tau_{-}}\right) \hat{g} R= & \frac{g_{-}}{2}\left(\frac{\hat{g} \hat{a}_{j} \hat{\Delta}_{s} \hat{n} \hat{\Delta}_{u}}{p_{-} \tau_{-}^{\prime}}+\frac{\hat{\Delta}_{s} \hat{n} \hat{\Delta}_{u} \hat{a}_{j} \hat{g}}{p_{-}^{\prime} \tau_{-}}\right)+g_{-}\left(\frac{\hat{a}_{j} \hat{\Delta}_{s} \hat{g}}{\tau_{-}^{\prime}}+\frac{\hat{g} \hat{\Delta}_{u} \hat{a}_{j}}{\tau_{-}}\right) \\
& +\left[\left(p_{-}^{\prime}+\tau_{-}^{\prime}\right)\left(g a_{j}\right)+g_{-}\left(\Delta_{s} a_{j}\right)\right] \frac{\hat{\Delta_{s}} \hat{n} \hat{g}}{p_{-}^{\prime} \tau_{-}^{\prime}}+\left[\left(p_{-}+\tau_{-}\right)\left(g a_{j}\right)+g_{-}\left(\Delta_{u} a_{j}\right)\right] \frac{\hat{g} \hat{n} \hat{\Delta}_{u}}{p_{-} \tau_{-}}-g^{2} \hat{a}_{j} \\
& -\left[\left(p_{-}^{\prime}+\tau_{-}^{\prime}\right) g^{2}+\frac{\tau_{-}^{\prime} g_{-}^{2}}{p_{-} \tau_{-}} \Delta_{u}^{2}\right] \frac{\hat{\Delta}_{s} \hat{n} \hat{a}_{j}}{2 p_{-}^{\prime} \tau_{-}^{\prime}}-\left[\left(p_{-}+\tau_{-}\right) g^{2}+\frac{\tau_{-} g_{-}^{2}}{p_{-}^{\prime} \tau_{-}^{\prime}} \Delta_{s}^{2}\right] \frac{\hat{a}_{j} \hat{n} \hat{\Delta}_{u}}{2 p_{-} \tau_{-}}+2\left(g a_{j}\right) \hat{g} \\
& -\left[g_{-} \Delta_{s}^{2}+2 p_{-}^{\prime}\left(g \Delta_{s}\right)\right] \frac{\hat{a}_{j} \hat{n} \hat{g}}{2 p_{-}^{\prime} \tau_{-}^{\prime}}-\left[g_{-} \Delta_{u}^{2}+2 p_{-}\left(g \Delta_{u}\right)\right] \frac{\hat{g} \hat{n} \hat{a}_{j}}{2 p_{-} \tau_{-}} \\
& +\frac{g_{-}}{p_{-} p_{-}^{\prime}}\left[\left(g a_{j}\right)+\frac{g_{-}}{\tau_{-}^{\prime}}\left(\Delta_{s} a_{j}\right)+\frac{g_{-}}{\tau_{-}}\left(\Delta_{u} a_{j}\right)\right] \hat{\Delta}_{s} \hat{n} \hat{\Delta}_{u}-g^{2}\left[\frac{\left(\Delta_{s} a_{j}\right)}{\tau_{-}^{\prime}}+\frac{\left(\Delta_{u} a_{j}\right)}{\tau_{-}}\right] \hat{n},
\end{aligned}
$$

and where it is clear that also in the case of $\Gamma_{R, \perp, j}\left(p, p^{\prime}, q ; \phi\right)$ the only term requiring regularization is the one analogous to that in the first line of Eq. (75). Due to the above change of variables, the various quantities appearing in $\Gamma_{R,-}\left(p, p^{\prime}, q ; \phi\right)$ and $\Gamma_{R, \perp, j}\left(p, p^{\prime}, q ; \phi\right)$ have to be interpreted as

$$
\begin{array}{lll}
\tau_{-}^{\prime}=z p_{-}^{\prime}-y q_{-}=(1-x-y) p_{-}^{\prime}-y q_{-}, & \pi_{s}^{\mu}=\pi_{p^{\prime}}^{\mu}\left(\theta_{S}^{\prime}\right), & \Delta_{s}^{\mu}=\mathcal{A}^{\mu}\left(\theta_{S}^{\prime}\right)-\mathcal{A}^{\mu}(\phi), \\
\tau_{-}=z p_{-}+x q_{-}=(1-x-y) p_{-}+x q_{-}, & \pi_{u}^{\mu}=\pi_{p}^{\mu}\left(\theta_{S}\right), & \Delta_{u}^{\mu}=\mathcal{A}^{\mu}\left(\theta_{S}\right)-\mathcal{A}^{\mu}(\phi),
\end{array}
$$

where

$$
\begin{aligned}
& \theta_{S}^{\prime}=\phi+2 x \tau_{-}^{\prime} S=\phi+2 x\left[(1-x-y) p_{-}^{\prime}-y q_{-}\right] S, \\
& \theta_{S}=\phi-2 y \tau_{-} S=\phi-2 y\left[(1-x-y) p_{-}+x q_{-}\right] S .
\end{aligned}
$$

The formal definitions of the other quantities such as $L, R, C^{\mu}, Q^{\lambda}$, and $Q^{\prime \lambda}$ remain unchanged, and the additional quantities

$$
g^{\mu}=\frac{G^{\mu}}{S}=x \int_{0}^{1} d \eta \pi_{p^{\prime}}^{\mu}\left(\theta_{\eta S}^{\prime}\right)+y \int_{0}^{1} d \eta \pi_{p}^{\mu}\left(\theta_{\eta S}\right)
$$

and

$$
\begin{aligned}
g_{1}^{\mu} & =\frac{G_{1}^{\mu}}{S^{2}}=\frac{d}{d \phi}\left[x^{2} \int_{0}^{1} d \eta \eta \pi_{p^{\prime}}^{\mu}\left(\theta_{\eta S}^{\prime}\right)-y^{2} \int_{0}^{1} d \eta \eta \pi_{p}^{\mu}\left(\theta_{\eta S}^{\prime}\right)\right] \\
& =\frac{x}{2 \tau_{-}^{\prime} S}\left[\pi_{p^{\prime}}^{\mu}\left(\theta_{S}^{\prime}\right)-\int_{0}^{1} d \eta \pi_{p^{\prime}}^{\mu}\left(\theta_{\eta S}^{\prime}\right)\right]+\frac{y}{2 \tau_{-} S}\left[\pi_{p}^{\mu}\left(\theta_{S}\right)-\int_{0}^{1} d \eta \pi_{p}^{\mu}\left(\theta_{\eta S}\right)\right],
\end{aligned}
$$

which is regular in the limit $S \rightarrow 0$ (and also in the limits $\tau_{-} \rightarrow 0$ and $\tau_{-}^{\prime} \rightarrow 0$ ), have also been introduced.

The above general expressions of the coefficients $\Gamma_{R,-}\left(p, p^{\prime}, q ; \phi\right)$ and $\Gamma_{R, \perp, j}\left(p, p^{\prime}, q ; \phi\right)$ give the possibility of constructing the amplitude $-i e \Gamma_{s, s^{\prime}, l}\left(p, p^{\prime}, q\right)$ [see Eq. (21)], which in turn can be used to compute, for example, the one-loop corrections to the basic QED processes in the presence of an arbitrary plane wave. The other building blocks of the one-loop corrections, the mass operator and the polarization operator, have been directly employed to compute elastic transition amplitudes and then the electron mass correction and the photon refractive indexes, respectively, in a general plane wave
$[66,67]$ or in a constant crossed field [72]. The vertex correction in vacuum has been used by Schwinger to compute the anomalous magnetic moment of the electron at the leading order in $\alpha$ [94]. The corresponding computation of the anomalous magnetic moment of the electron in the presence of an arbitrary plane wave by starting from the vertex correction obtained above features a difficulty due to the fact that physically the electron interacts with the total magnetic field, given here by the magnetic field of the external photon and the magnetic field of the plane wave. Indeed, the magnetic field of the plane wave is by assumption much stronger than that of the external photon such that it is much more convenient to compute the 
anomalous magnetic moment of the electron directly only in the presence of the plane wave. This is indeed what has already been done in the case of a constant crossed field $[72,95]$ by starting from the dressed mass operator and by computing the spin-dependent electron mass shift (note that although the vertex correction in a constant crossed field is computed in Refs. [78,79], the anomalous magnetic moment of the electron is not mentioned in those papers). In the case of a general plane wave, due to the time dependence of the latter, it is not possible to introduce a (constant) anomalous magnetic moment of the electron. Nevertheless, the possibility of measuring spin-dependent radiative corrections in an ultrashort laser pulse has been studied [96] (see also Ref. [97]).

\section{THE LOCALLY CONSTANT FIELD APPROXIMATION}

In this section, we would like to investigate the regularized vertex-correction function $\Gamma_{R}^{\mu}\left(p, p^{\prime}, q ; \phi\right)$ in the LCFA
$[12,15,24,50]$. Under this approximation quantum processes in an external field are assumed to form over a length much shorter than the typical length where the external field significantly varies $[12,15,24,50]$. As a general condition of validity of the LCFA in a plane wave, one assumes that the strength of the vector potential of the plane wave times the elementary charge is much larger than the electron mass. This condition is based on the idea that the strength of the vector potential scales as the strength of the electric field of the wave times the typical field wavelength, and that the LCFA applies for larger and larger wavelengths (see Refs. [39,45,47-49,68,89,90,98-102] for more refined results and investigations about the validity of the LCFA). In order to study the structure of the vertex-correction function $\Gamma_{R}^{\mu}\left(p, p^{\prime}, q ; \phi\right)$, it is first useful to exploit the general structure of the external plane wave, in particular, to rewrite the phase $G^{2} / S$ in a convenient form [see Eqs. (50) and (61)]. By starting from the identity $v^{2}=2 v_{+} v_{-}-v_{\perp}^{2}$, valid for a generic four-vector $v^{\mu}$, it can easily be shown that

$$
\begin{aligned}
G^{2}= & \frac{s\left(p_{-}^{\prime} s+p_{-} u\right)}{p_{-}^{\prime}}\left(m^{2}+\delta m_{s}^{2}\right)+\frac{u\left(p_{-}^{\prime} s+p_{-} u\right)}{p_{-}}\left(m^{2}+\delta m_{u}^{2}\right) \\
& +u s p_{-} p_{-}^{\prime}\left\{\frac{1}{p_{-}^{\prime}}\left[\boldsymbol{\pi}_{p^{\prime}, \perp}(\phi)-\frac{1}{s} \int_{0}^{s} d s^{\prime} \boldsymbol{\Delta}_{s^{\prime}, \perp}\right]-\frac{1}{p_{-}}\left[\boldsymbol{\pi}_{p, \perp}(\phi)-\frac{1}{u} \int_{0}^{u} d u^{\prime} \boldsymbol{\Delta}_{u^{\prime}, \perp}\right]\right\}^{2},
\end{aligned}
$$

where we have introduced the laser-induced square mass corrections

$$
\begin{gathered}
\delta m_{s}^{2}=\frac{1}{s} \int_{0}^{s} d s^{\prime} \mathcal{A}_{\perp}^{2}\left(\psi_{s^{\prime}}\right)-\frac{1}{s^{2}}\left[\int_{0}^{s} d s^{\prime} \mathcal{A}_{\perp}\left(\psi_{s^{\prime}}\right)\right]^{2}=\frac{1}{s} \int_{0}^{s} d s^{\prime} \boldsymbol{\Delta}_{s^{\prime}, \perp}^{2}-\frac{1}{s^{2}}\left(\int_{0}^{s} d s^{\prime} \boldsymbol{\Delta}_{s^{\prime}, \perp}\right)^{2}, \\
\delta m_{u}^{2}=\frac{1}{u} \int_{0}^{u} d u^{\prime} \mathcal{A}_{\perp}^{2}\left(\psi_{u^{\prime}}\right)-\frac{1}{u^{2}}\left[\int_{0}^{u} d u^{\prime} \mathcal{A}_{\perp}\left(\psi_{u^{\prime}}\right)\right]^{2}=\frac{1}{u} \int_{0}^{u} d u^{\prime} \boldsymbol{\Delta}_{u^{\prime}, \perp}^{2}-\frac{1}{u^{2}}\left(\int_{0}^{u} d u^{\prime} \boldsymbol{\Delta}_{u^{\prime}, \perp}\right)^{2} .
\end{gathered}
$$

We notice that for the present case of on-shell electrons, the quantity $G^{2}$ is non-negative, a property which will be used below. Also, we observe that for the evaluation of $\Gamma_{R}^{\mu}\left(p, p^{\prime}, q ; \phi\right)$ the phase $\phi$ is fixed but the vector potential depends on the integration variables $s, u$, and $t$ (or $x, y$, and $S$ ). Thus, within the integration region, the terms in the phases depending on the vector potential become larger and larger, leading in turn to highly oscillating integrands. Thus, the largest contributions to the integrals in the proper times come from the regions where these variables are sufficiently small that the squares of the mass corrections $\delta m_{s}^{2}$ and $\delta m_{u}^{2}$ are of the order of $m^{2}$ [70] (see also Ref. [103] for a study of the subleading contributions arising from the saddle points of the phases). In order to implement this idea, we assume that the variables $s$ and $u$ in Eqs. (87) and (88) in the regions mainly contributing to the corresponding integrals are sufficiently small to expand the integrands in those equations for $\psi_{s^{\prime}}$ and $\psi_{u^{\prime}}$ around $\phi$ (the validity of this assumption is checked a posteriori). It is appropriate to perform the expansions up to terms proportional to the second derivative of $\mathcal{A}_{\perp}(\phi)$ because the leading-order contributions to $\delta m_{s}^{2}$ and to $\delta m_{u}^{2}$ turn out to be proportional to $\mathcal{A}_{\perp}^{\prime 2}(\phi)$, i.e., to the square of the first-order correction. Indeed, all contributions proportional to $\mathcal{A}_{\perp}^{\prime \prime}(\phi)$ cancel out, and one obtains

$$
\begin{aligned}
& \delta m_{s}^{2} \approx \frac{1}{3} m^{2}\left[\frac{t \chi_{p^{\prime}}(\phi)-u \chi_{q}(\phi)}{S}\right]^{2} m^{4} s^{2}, \\
& \delta m_{u}^{2} \approx \frac{1}{3} m^{2}\left[\frac{t \chi_{p}(\phi)+s \chi_{q}(\phi)}{S}\right]^{2} m^{4} u^{2},
\end{aligned}
$$

where $\chi_{p}(\phi)=p_{-}\left|\mathcal{E}_{\perp}(\phi)\right| / m^{3}, \chi_{p^{\prime}}(\phi)=p_{-}^{\prime}\left|\mathcal{E}_{\perp}(\phi)\right| / m^{3}$, and $\quad \chi_{q}(\phi)=q_{-}\left|\mathcal{E}_{\perp}(\phi)\right| / m^{3}=\chi_{p}(\phi)-\chi_{p^{\prime}}(\phi)$, with $\mathcal{E}_{\perp}(\phi)=-\mathcal{A}_{\perp}^{\prime}(\phi)$ [recall that we have assumed that $q_{-}>0$, such that $\left.\chi_{q}(\phi) \geq 0\right]$. Now, in order to obtain the range of validity of the above approximations, it is easier to consider the typical situation in which $p_{-} \sim p_{-}^{\prime} \sim q_{-}$and to 
indicate as $p_{0,-}$ this common light-cone energy scale. Correspondingly, by indicating as $E_{0}$ and $\omega_{0}$ the amplitude and the typical angular frequency of the background plane wave $\left(\omega_{0}\right.$ can also be thought of as the inverse of the typical time interval over which the background field varies significantly), we construct the well-known Lorentz- and gauge-invariant parameters $\xi_{0}=\mathcal{E}_{0} / m \omega_{0}, \chi_{0}=p_{0,-} \mathcal{E}_{0} / m^{3}$, and $\eta_{0}=\chi_{0} / \xi_{0}=\omega_{0} p_{0,-} / m^{2}[12,15,24]$, with $\mathcal{E}_{0}=|e| E_{0}$. The above approximations are all valid if the integrals are formed over regions of $s$ and $u$ such that $\omega_{0} s p_{0,-}=$ $m^{2} s \eta_{0} \ll 1$ and $\omega_{0} u p_{0,-}=m^{2} u \eta_{0} \ll 1$ (note that the additional proper time variable $t$ appears in the equations in a way that the relevant conditions and estimates do not involve it). Now, from the expressions of the mass corrections within the LCFA, it is easily seen that if $\chi_{0} \sim 1$ $\left(\chi_{0} \gg 1\right)$, then the integrals are formed over the region where $s, u \lesssim 1 / m^{2}\left(s, u \lesssim 1 / \chi_{0}^{2 / 3} m^{2}\right)$. Since here we are interested in situations where $\chi_{0} \gtrsim 1$, we can for simplicity use the single expression $s, u \lesssim 1 / \chi_{0}^{2 / 3} m^{2}$, such that the LCFA is valid if $\eta_{0} / \chi_{0}^{2 / 3}=\chi_{0}^{1 / 3} / \xi_{0} \ll 1$, together with the original assumption about the product of the laser electric field amplitude and the laser wavelength being large, which corresponds to the condition $\xi_{0} \gg 1[45,48,49,68,89,90$, $98,99,101]$. By also expanding the terms in the second line of Eq. (86) up to the second derivative of $\boldsymbol{A}_{\perp}(\phi)$, we obtain that at the leading order in the LCFA the phase $G^{2} / S$ reads

$$
\begin{aligned}
\frac{G^{2}}{S}= & g^{2} S \approx \frac{p_{-}^{\prime} s+p_{-} u}{p_{-}^{\prime} S} m^{2} s\left\{1+\frac{1}{3}\left[\frac{t \chi_{p^{\prime}}(\phi)-u \chi_{q}(\phi)}{S}\right]^{2} m^{4} s^{2}\right\}+\frac{p_{-}^{\prime} s+p_{-} u}{p_{-} S} m^{2} u\left\{1+\frac{1}{3}\left[\frac{t \chi_{p}(\phi)+s \chi_{q}(\phi)}{S}\right]^{2} m^{4} u^{2}\right\} \\
& +\frac{u s p_{-} p_{-}^{\prime}}{S}\left\{\frac{1}{p_{-}^{\prime}}\left[\boldsymbol{p}_{\perp}^{\prime}-\mathcal{A}_{\perp}(\phi)+m^{3} s \frac{t \chi_{\perp, p^{\prime}}(\phi)-u \chi_{\perp, q}(\phi)}{S}\right]\right. \\
& \left.-\frac{1}{p_{-}}\left[\boldsymbol{p}_{\perp}-\mathcal{A}_{\perp}(\phi)-m^{3} u \frac{t \chi_{\perp, p}(\phi)+s \chi_{\perp, q}(\phi)}{S}\right]\right\}^{2}+\frac{4}{3} \frac{u s p_{-} p_{-}^{\prime}}{S}\left(\frac{s^{2} \tau_{-}^{\prime 2}}{p_{-}^{\prime}}-\frac{u^{2} \tau_{-}^{2}}{p_{-}}\right) \mathcal{E}_{\perp}^{\prime}(\phi) \cdot \mathcal{V}_{\perp}(\phi),
\end{aligned}
$$

where $\quad \chi_{\perp, p}(\phi)=p_{-} \mathcal{E}_{\perp}(\phi) / m^{3} \quad\left[\chi_{p}(\phi)=\left|\chi_{\perp, p}(\phi)\right|\right], \quad \chi_{\perp, p^{\prime}}(\phi)=p_{-}^{\prime} \mathcal{E}_{\perp}(\phi) / m^{3} \quad\left[\chi_{p^{\prime}}(\phi)=\left|\chi_{\perp, p^{\prime}}(\phi)\right|\right], \quad \chi_{\perp, q}(\phi)=$ $q_{-} \mathcal{E}_{\perp}(\phi) / m^{3}=\chi_{\perp, p}(\phi)-\chi_{\perp, p^{\prime}}(\phi)\left[\chi_{q}(\phi)=\left|\chi_{\perp, q}(\phi)\right|\right]$, and where

$$
\mathcal{V}_{\perp}(\phi)=\frac{1}{p_{-}^{\prime}}\left[p_{\perp}^{\prime}-\mathcal{A}_{\perp}(\phi)\right]-\frac{1}{p_{-}}\left[\boldsymbol{p}_{\perp}-\mathcal{A}_{\perp}(\phi)\right]
$$

The appearance of $\mathcal{A}_{\perp}(\phi)$ in the vector $\mathcal{V}_{\perp}(\phi)$ seems to indicate that indeed the last line of Eq. (90) is leading order in the LCFA because $\left|\mathcal{V}_{\perp}(\phi)\right|$ scales as $1 / \omega_{0}$. We should, however, recall that the final object to be computed is $\Gamma_{s, s^{\prime}, l}\left(p, p^{\prime}, q\right)$ in Eq. (21). Now, if we compute the phase $\Phi\left(p, p^{\prime}, q ; \phi\right)$ resulting from the functions in Eq. (21) other than $\Gamma^{\mu}\left(p, p^{\prime}, q ; \phi\right)$, after taking the integrals in $\boldsymbol{x}_{\perp}$ and $T$, we obtain (apart from an inessential constant)

$$
\Phi\left(p, p^{\prime}, q ; \phi\right)=\frac{q_{-}}{2 p_{-} p_{-}^{\prime}} \int_{0}^{\phi} d \phi^{\prime}\left\{m^{2}+\frac{p_{-} p_{-}^{\prime}}{q_{-}^{2}} q^{2}+\left[\boldsymbol{p}_{\perp}-\mathcal{A}_{\perp}\left(\phi^{\prime}\right)-\frac{p_{-}}{q_{-}} \boldsymbol{q}_{\perp}\right]^{2}\right\}
$$

together with the conservation laws $\boldsymbol{p}_{\perp}=\boldsymbol{p}_{\perp}^{\prime}+\boldsymbol{q}_{\perp}$ and $p_{-}=p_{-}^{\prime}+q_{-}$. It is clear that, apart from the term proportional to $q^{2}$, this is the phase of nonlinear Compton scattering, as given, e.g., in Ref. [45]. By using the conservation laws $\boldsymbol{p}_{\perp}=$ $\boldsymbol{p}_{\perp}^{\prime}+\boldsymbol{q}_{\perp}$ and $p_{-}=p_{-}^{\prime}+q_{-}$, it is easy to show that

$$
\mathcal{V}_{\perp}(\phi)=\frac{q_{-}}{p_{-} p_{-}^{\prime}}\left[\boldsymbol{p}_{\perp}-\mathcal{A}_{\perp}(\phi)-\frac{p_{-}}{q_{-}} \boldsymbol{q}_{\perp}\right] .
$$

Since the LCFA corresponds to evaluate the remaining integral in $\phi$ in Eq. (21), it is clear that in the region where most of the photons are emitted (and tacitly assuming that the virtuality $q^{2}$ is less than or of the order of $m^{2}$ ), it is $\left|\mathcal{V}_{\perp}(\phi)\right| \sim m$ and the last term in Eq. (90) can be neglected. Finally, by applying the changes of variables discussed above, we obtain the final expression of $g^{2} S$ within the LCFA in the form

$$
\begin{aligned}
g^{2} S \approx & \frac{p_{-}^{\prime} x+p_{-} y}{p_{-}^{\prime}} x m^{2} S\left\{1+\frac{1}{3}\left[z \chi_{p^{\prime}}(\phi)-y \chi_{q}(\phi)\right]^{2} x^{2} m^{4} S^{2}\right\}+\frac{p_{-}^{\prime} x+p_{-} y}{p_{-}} y m^{2} S\left\{1+\frac{1}{3}\left[z \chi_{p}(\phi)+x \chi_{q}(\phi)\right]^{2} y^{2} m^{4} S^{2}\right\} \\
& +x y p_{-} p_{-}^{\prime} S\left\{\mathcal{V}_{\perp}(\phi)+m^{3} S\left[\frac{x z}{p_{-}^{\prime}} \chi_{\perp, p^{\prime}}(\phi)+\frac{y z}{p_{-}} \chi_{\perp, p}(\phi)-\frac{x y q_{-}}{p_{-} p_{-}^{\prime}} \chi_{\perp, q}(\phi)\right]\right\}^{2},
\end{aligned}
$$

where $z=1-x-y$. 
Finally, we point out that we have explicitly proved that in the constant-crossed field case $\mathcal{A}_{\perp}(\phi)=-\mathcal{E}_{0} \phi$, the phase $g^{2} S$ reduces to the phase computed in Refs. [78,79]. The same can be verified starting from Eq. (94) by setting $\mathcal{E}_{\perp}(\phi)=\mathcal{E}_{0}$, and we note that our expression of the phase of the vertex-correction function is not only more general but also much more compact than that presented in Refs. [78,79]. A comparison of the final expression of the preexponent was not carried out as the form presented in Refs. $[78,79]$ has a very different structure from ours, due to employed transformations there, which are appropriate only to the constant-crossed field case. It is interesting to notice that while the mass operator and the polarization operator in a constant crossed field can be expressed in terms of Airy and Scorer functions [72,73], this is not the case for the vertex correction $[78,79]$, which also occurs here within the LCFA. The reason is that in the case of the mass operator and of the polarization operator, the phase of the whole integrand depends on the variable corresponding to the sum of the proper times only via linear and cubic terms, whose integral indeed results in Airy and Scorer functions. In the case of the vertex correction, a quadratic term also appears [see Eq. (45) in both Refs. [78,79] and the dependence on $S$ of the last line of Eq. (94)], which prevents one from taking the integral analytically (note that a shift of the variable to remove the quadratic term would not help as the integral is from 0 to $\infty$ ). By comparing the vertex correction with the mass operator, which also features two external fermions, one curiously notices that if in the former case one equals the transverse momenta and the light-cone energy of the two fermions $\left(\boldsymbol{p}_{\perp}^{\prime}=\boldsymbol{p}_{\perp}\right.$ and $p_{-}^{\prime}=p_{-}$here), which is always the case in the massoperator case, one indeed obtains that the quadratic terms in the sum of the proper times in the phase vanish [see Eq. (45) in both Refs. [78,79] and the last line of Eq. (94)].

Passing now to the preexponents of the components $\Gamma_{R,-}\left(p, p^{\prime}, q ; \phi\right)$ [see Eq. (75)] and $\Gamma_{R, \perp, j}\left(p, p^{\prime}, q ; \phi\right)$ [see Eq. (76)], one has to expand the field-dependent terms in Eqs. (75) and (76) around the phase $\phi$. Taking into account that the final quantity to be evaluated is $-i e \Gamma_{s, s^{\prime}, l}\left(p, p^{\prime}, q\right)$ in Eq. (21), a lengthy but straightforward calculation shows that

$$
\begin{aligned}
\Gamma_{R,-}\left(p, p^{\prime}, q ; \phi\right) \approx & \frac{\alpha}{2 \pi} \hat{n} \int_{0}^{\infty} \frac{d S}{S} \int_{\delta} d x d y d z e^{-i \kappa^{2} z S}\left[e^{-i g^{2} S}-e^{-i m^{2}(x+y)^{2} S}\right] \\
& -\frac{i \alpha}{2 \pi} \int_{0}^{\infty} d S \int_{\delta} d x d y d z e^{-i \kappa^{2} z S}\left\langlee ^ { - i g ^ { 2 } S } \left\{\left(p_{-}+p_{-}^{\prime}-g_{-}\right)\left(\frac{1-x}{p_{-}^{\prime}}+\frac{1-y}{p_{-}}\right) m^{2} \hat{n}-2 m^{2} \hat{n}\right.\right. \\
& +2 m\left[\left(p_{-}+p_{-}^{\prime}-g_{-}\right)(x+y)-g_{-}\right]+\left[(2-x)\left(p_{-} b^{\prime}+p_{-}^{\prime} b\right)-g_{-} b\right]\left[(2-y)\left(p_{-} b^{\prime}+p_{-}^{\prime} b\right)-g_{-} b^{\prime}\right] \frac{\mathcal{A}_{\perp}^{\prime 2}}{p_{-} p_{-}^{\prime}} \hat{n} \\
& +(1-x)(1-y) p_{-} p_{-}^{\prime} \mathcal{V}_{\perp}^{2} \hat{n}-2(1-x)(1-y) p_{-} p_{-}^{\prime}\left(\frac{2-x}{1-x} \frac{b^{\prime}}{p_{-}^{\prime}}+\frac{2-y}{1-y} \frac{b}{p_{-}}\right) \mathcal{V}_{\perp} \cdot \mathcal{A}_{\perp}^{\prime} \hat{n} \\
& +2 m\left[g_{-}-\left(p_{-}+p_{-}^{\prime}-g_{-}\right)(x+y)\right]\left(\frac{b^{\prime}}{p_{-}^{\prime}}+\frac{b}{p_{-}}\right) \hat{n} \hat{\mathcal{A}}^{\prime} \\
& \left.-\left(p_{-}+p_{-}^{\prime}-g_{-}\right)\left[x \frac{b^{\prime}}{p_{-}^{\prime}} \hat{\mathcal{A}}^{\prime} \hat{n}\left(\frac{q_{-}}{p_{-}} m+p_{-}^{\prime} \gamma_{\perp} \cdot \mathcal{V}_{\perp}\right)+y \frac{b}{p_{-}}\left(\frac{q_{-}}{p_{-}^{\prime}} m+p_{-} \gamma_{\perp} \cdot \mathcal{V}_{\perp}\right) \hat{n} \hat{\mathcal{A}}^{\prime}\right]\right\} \\
& \left.-m^{2} \hat{n} e^{-i m^{2}(x+y)^{2} S}\left[2 z-(x+y)^{2}\right]\right\rangle
\end{aligned}
$$

where $g^{2}$ is obtained from Eq. (94), $b=y \tau_{-} S, b^{\prime}=x \tau_{-}^{\prime} S$, and where all fields and derivatives are evaluated at $\phi$.

Analogously, one obtains the following expression for $\Gamma_{R, \perp, j}\left(p, p^{\prime}, q ; \phi\right)$ within the LCFA:

$$
\begin{aligned}
\Gamma_{R, \perp, j}\left(p, p^{\prime}, q ; \phi\right) \approx & -\frac{\alpha}{2 \pi} \hat{a}_{j} \int_{0}^{\infty} \frac{d S}{S} \int_{\delta} d x d y d z e^{-i \kappa^{2} z S}\left[e^{-i g^{2} S}-e^{-i m^{2}(x+y)^{2} S}\right] \\
& +\frac{i \alpha}{2 \pi} \int_{0}^{\infty} d S \int_{\delta} d x d y d z e^{-i \kappa^{2} z S}\left(e ^ { - i g ^ { 2 } S } \left\langle2\left[\left(\pi_{s} \pi_{u}\right)-\left(\left(\pi_{s}+\pi_{u}\right) g\right)\right]\left(\hat{a}_{j}+\frac{g_{-} b^{\prime}}{p_{-}^{\prime} \tau_{-}^{\prime}} \hat{n} \hat{\mathcal{A}}^{\prime} \hat{a}_{j}+\frac{g_{-} b}{p_{-} \tau_{-}} \hat{a}_{j} \hat{n} \hat{\mathcal{A}}^{\prime}\right)\right.\right. \\
& +\frac{i}{S}\left(\frac{g_{-} b^{\prime}}{p_{-}^{\prime} \tau_{-}^{\prime}} \hat{n} \hat{\mathcal{A}}^{\prime} \hat{a}_{j}+\frac{g_{-} b}{p_{-} \tau_{-}} \hat{a}_{j} \hat{n} \hat{\mathcal{A}}^{\prime}\right)+L\left(C a_{j}\right) \hat{\pi}_{s} \hat{g} R+L \hat{g} \hat{\pi}_{u}\left(C a_{j}\right) R \\
& -L \hat{g}\left(\hat{a}_{j}+\frac{b^{\prime}}{\tau_{-}^{\prime}} \hat{a}_{j} \hat{\mathcal{A}}^{\prime} \hat{n}+\frac{b}{\tau_{-}} \hat{\mathcal{A}}^{\prime} \hat{n} \hat{a}_{j}\right) \hat{g} R-2 S\left(g g_{1}\right)\left\{\left[\frac{2 b^{\prime} \tau_{-}}{p_{-}^{\prime}}+(2-y) b+x b^{\prime}\right] \hat{n} \hat{\mathcal{A}}^{\prime} \hat{a}_{j}\right.
\end{aligned}
$$




$$
\begin{aligned}
& +\left[\frac{2 b \tau_{-}^{\prime}}{p_{-}}+(2-x) b^{\prime}+y b\right] \hat{a}_{j} \hat{n} \hat{\mathcal{A}}^{\prime}+m\left[\frac{p_{-}^{\prime}}{p_{-}}-\frac{p_{-}}{p_{-}^{\prime}}+x\left(1-\frac{p_{-}^{\prime}}{p_{-}}\right)-y\left(1-\frac{p_{-}}{p_{-}^{\prime}}\right)\right] \hat{n} \hat{a}_{j} \\
& \left.-p_{-}(1-y) \gamma_{\perp} \cdot \mathcal{V}_{\perp} \hat{n} \hat{a}_{j}-p_{-}^{\prime}(1-x) \hat{n} \hat{a}_{j} \gamma_{\perp} \cdot \mathcal{V}_{\perp}\right\}+i(x-y)(x+y-2) \mathcal{A}_{\perp}^{\prime} \cdot \boldsymbol{a}_{j} \hat{n} \\
& \left.\left.+i\left[x-(y+z) \frac{\tau_{-}}{p_{-}^{\prime}}\right] \hat{\mathcal{A}}^{\prime} \hat{n} \hat{a}_{j}-i\left[y-(x+z) \frac{\tau_{-}^{\prime}}{p_{-}}\right] \hat{a}_{j} \hat{n} \hat{\mathcal{A}}^{\prime}\right\rangle-m^{2} \hat{a}_{j} e^{-i m^{2}(x+y)^{2} S}\left[2 z-(x+y)^{2}\right]\right),
\end{aligned}
$$

where

$$
\begin{aligned}
& \left(\pi_{s} \pi_{u}\right)-\left(\left(\pi_{s}+\pi_{u}\right) g\right) \approx-\frac{p_{-}+p_{-}^{\prime}}{2 g_{-}} g^{2}-\frac{g_{-}}{p_{-}+p_{-}^{\prime}} m^{2} \\
& +\frac{1}{2}\left(1-\frac{g_{-}}{p_{-}+p_{-}^{\prime}}\right)\left\{\left(\frac{p_{-}}{p_{-}^{\prime}}+\frac{p_{-}^{\prime}}{p_{-}}\right) m^{2}+\left[\mathcal{V}_{\perp}-2\left(\frac{b^{\prime}}{p_{-}^{\prime}}+\frac{b}{p_{-}}\right) \mathcal{A}_{\perp}^{\prime}\right]^{2} p_{-} p_{-}^{\prime}\right\} \\
& -\frac{p_{-}^{2} p_{-}^{\prime 2}}{2 g_{-}\left(p_{-}+p_{-}^{\prime}\right)}\left[(x-y) \mathcal{V}_{\perp}-\frac{\left(x b^{\prime}+y b\right) q_{-}+2 x b p_{-}^{\prime}-2 y b^{\prime} p_{-}}{p_{-} p_{-}^{\prime}} \mathcal{A}_{\perp}^{\prime}\right]^{2}, \\
& L\left(C a_{j}\right) \hat{\pi}_{s} \hat{g} R+L \hat{g} \hat{\pi}_{u}\left(C a_{j}\right) R-L \hat{g}\left(\hat{a}_{j}+\frac{b^{\prime}}{\tau_{-}^{\prime}} \hat{a}_{j} \hat{\mathcal{A}}^{\prime} \hat{n}+\frac{b}{\tau_{-}} \hat{\mathcal{A}}^{\prime} \hat{n} \hat{a}_{j}\right) \hat{g} R \\
& \approx g^{2}\left(\hat{a}_{j}+\frac{g_{-} b^{\prime}}{p_{-}^{\prime} \tau_{-}^{\prime}} \hat{n} \hat{\mathcal{A}}^{\prime} \hat{a}_{j}+\frac{g_{-} b}{p_{-} \tau_{-}} \hat{a}_{j} \hat{n} \hat{\mathcal{A}}^{\prime}\right)+2\left[\left(\left(\pi_{s}+\pi_{u}-g\right) a_{j}\right)\right. \\
& \left.-S\left(p_{-}+p_{-}^{\prime}-g_{-}\right)(x-y) \mathcal{A}_{\perp}^{\prime} \cdot \boldsymbol{a}_{j}\right] L \hat{g} R-2 m\left(g a_{j}\right)\left[1-\left(\frac{b^{\prime}}{p_{-}^{\prime}}+\frac{b}{p_{-}}\right) S \hat{n} \hat{\mathcal{A}}^{\prime}\right] \\
& -m S\left(x \hat{a}_{j} \hat{\mathcal{A}}^{\prime}-y \hat{\mathcal{A}}^{\prime} \hat{a}_{j}\right) \hat{n}\left[\left(b y-b^{\prime} x-2 \frac{b g_{-}}{p_{-}}\right) \hat{\mathcal{A}}^{\prime}+m\left(y+x \frac{p_{-}^{\prime}}{p_{-}}\right)-x p_{-}^{\prime} \gamma_{\perp} \cdot \mathcal{V}_{\perp}\right] \\
& -m S\left[\left(b y-b^{\prime} x+2 \frac{b^{\prime} g_{-}}{p_{-}^{\prime}}\right) \hat{\mathcal{A}}^{\prime}+m\left(x+y \frac{p_{-}}{p_{-}^{\prime}}\right)+y p_{-} \gamma_{\perp} \cdot \mathcal{V}_{\perp}\right] \hat{n}\left(x \hat{a}_{j} \hat{\mathcal{A}}^{\prime}-y \hat{\mathcal{A}}^{\prime} \hat{a}_{j}\right) \\
& -2 i\left(p_{-}+p_{-}^{\prime}-g_{-}\right)(x+y) S \varepsilon^{\lambda \mu \nu \rho} n_{\lambda} \mathcal{A}_{\mu}^{\prime} a_{j, \nu} \tilde{n}_{\rho} \gamma^{5} L \hat{g} R, \\
& L \hat{g} R \approx m(x+y)\left[1-\left(\frac{b}{p_{-}}+\frac{b^{\prime}}{p_{-}^{\prime}}\right) \hat{n} \hat{\mathcal{A}}^{\prime}\right]-\frac{x b^{\prime}}{2 p_{-}^{\prime}} \hat{\mathcal{A}}^{\prime} \hat{n}\left(m \frac{q_{-}}{p_{-}}+p_{-}^{\prime} \mathcal{V}_{\perp} \cdot \gamma_{\perp}\right) \\
& -\frac{y b}{2 p_{-}}\left(m \frac{q_{-}}{p_{-}^{\prime}}+p_{-} \mathcal{V}_{\perp} \cdot \gamma_{\perp}\right) \hat{n} \hat{\mathcal{A}}^{\prime}-\left(\frac{1}{3} \frac{x b^{\prime 2}}{p_{-}^{\prime}}+\frac{1}{3} \frac{y b^{2}}{p_{-}}+\frac{g_{-} b b^{\prime}}{p_{-} p_{-}^{\prime}}\right) \hat{n} \mathcal{A}_{\perp}^{\prime 2}, \\
& \gamma^{5} L \hat{g} R \approx \gamma^{5}\left[m(y-x)+m\left(\frac{x b}{p_{-}}-\frac{y b^{\prime}}{p_{-}^{\prime}}\right) \hat{n} \hat{\mathcal{A}}^{\prime}-\frac{x b^{\prime}}{2 p_{-}^{\prime}} \hat{\mathcal{A}}^{\prime} \hat{n}\left(m \frac{q_{-}}{p_{-}}+p_{-}^{\prime} \mathcal{V}_{\perp} \cdot \gamma_{\perp}\right)\right. \\
& \left.+\frac{y b}{2 p_{-}}\left(m \frac{q_{-}}{p_{-}^{\prime}}-p_{-} \mathcal{V}_{\perp} \cdot \gamma_{\perp}\right) \hat{n} \hat{\mathcal{A}}^{\prime}-\left(\frac{1}{3} \frac{x b^{\prime 2}}{p_{-}^{\prime}}+\frac{1}{3} \frac{y b^{2}}{p_{-}}+\frac{g_{-} b b^{\prime}}{p_{-} p_{-}^{\prime}}\right) \hat{n} \mathcal{A}_{\perp}^{\prime 2}\right], \\
& \left(\left(\pi_{s}+\pi_{u}\right) a_{j}\right) \approx-\left[2\left(b-b^{\prime}\right) \mathcal{A}_{\perp}^{\prime}+\frac{p_{-}+p_{-}^{\prime}}{q_{-}} \boldsymbol{q}_{\perp}+2 \frac{p_{-} p_{-}^{\prime}}{q_{-}} \mathcal{V}_{\perp}\right] \cdot \boldsymbol{a}_{j}, \\
& \left(g a_{j}\right) \approx\left[\left(x b^{\prime}-y b\right) \mathcal{A}_{\perp}^{\prime}-\left(y p_{-}+x p_{-}^{\prime}\right) \frac{\boldsymbol{q}_{\perp}}{q_{-}}-(x+y) \frac{p_{-} p_{-}^{\prime}}{q_{-}} \mathcal{V}_{\perp}\right] \cdot \boldsymbol{a}_{j}, \\
& \left(g g_{1}\right) \approx\left[\frac{x^{3} b^{\prime}}{6}+\frac{y^{3} b}{6}+x y^{2} p_{-}^{\prime}\left(\frac{2 b}{3 p_{-}}+\frac{b^{\prime}}{2 p_{-}^{\prime}}\right)+y x^{2} p_{-}\left(\frac{2 b^{\prime}}{3 p_{-}^{\prime}}+\frac{b}{2 p_{-}}\right)\right] \mathcal{A}_{\perp}^{\prime 2} \\
& -\frac{x y}{2}\left(y p_{-}^{\prime}+x p_{-}\right) \mathcal{V}_{\perp} \cdot \mathcal{A}_{\perp}^{\prime},
\end{aligned}
$$


where $\gamma^{5}=i \gamma^{0} \gamma^{1} \gamma^{2} \gamma^{3}$ and $\varepsilon^{\mu \nu \lambda \rho}$ is the completely antisymmetric tensor with $\varepsilon^{0123}=+1$. In the above equations, the quantity $g^{2}$ is given in Eq. (94), and we have taken into account that finally we need the matrix elements of these matrices between $\bar{U}_{s^{\prime}}\left(p^{\prime}, x\right)$ and $U_{s}(p, x)$. The appearance of $\boldsymbol{q}_{\perp}$ in Eqs. (101) and (102) may suggest that large terms (i.e., of the order of $1 / \omega_{0}$ ) could appear in total probabilities, if one imagines to carry out the integral over the transverse photon momentum, as one has to shift the variable $\boldsymbol{q}_{\perp}$ by a vector containing $\mathcal{A}_{\perp}(\phi)$ [see Eq. (92)] in order to perform the resulting Gaussian integral. However, this does not represent a problem because the vector $\boldsymbol{q}_{\perp}$ is always multiplied by $\boldsymbol{a}_{j}$ [see Eqs. (101) and (102)]. Indeed, the first equality in Eq. (45) shows that the components $\Gamma_{R, \perp, j}\left(p, p^{\prime}, q ; \phi\right)$ are only auxiliary quantities, as one finally needs to compute the components $\left(\Gamma_{R}\left(p, p^{\prime}, q ; \phi\right) \Lambda_{j}\right)$. Since by definition the vector $\boldsymbol{\Lambda}_{\perp, j}$ is perpendicular to $\boldsymbol{q}_{\perp}$ [see Eq. (42)], by replacing $a_{j}^{\mu}$ with $\Lambda_{j}^{\mu}$ in $\Gamma_{R, \perp, j}\left(p, p^{\prime}, q ; \phi\right)$, it is easy to see that $\left(\Gamma_{R}\left(p, p^{\prime}, q ; \phi\right) \Lambda_{j}\right)$ depends on the vector $\boldsymbol{q}_{\perp}$ only through $\boldsymbol{\Lambda}_{\perp, j}$, a quantity which then, due to completeness, drops out once one computes total probabilities.

We recall that the LCFA is a useful approximation also because the corresponding tree-level probabilities of the basic strong-field QED processes, nonlinear Compton scattering and nonlinear Breit-Wheeler pair production, are widely employed in numerical codes used to study, for example, the evolution of QED cascades [104-110]. The components $\Gamma_{R,-}\left(p, p^{\prime}, q ; \phi\right)$ and $\Gamma_{R, \perp, j}\left(p, p^{\prime}, q ; \phi\right)$ in Eqs. (95) and (96), although being evaluated within the LCFA, cannot by themselves be implemented in numerical codes (for example, they would diverge in the physical limit of massless photons). A possibility in that direction would be if one would need more accurate probabilities of nonlinear Compton scattering and nonlinear Breit-Wheeler pair production, which includes one-loop radiative corrections. In this case, the components $\Gamma_{R,-}\left(p, p^{\prime}, q ; \phi\right)$ and $\Gamma_{R, \perp, j}\left(p, p^{\prime}, q ; \phi\right)$ in Eqs. (95) and (96) represent, together with the mass operator and with the polarization operator, the necessary starting point to evaluate the one-loop differential probabilities of those processes. Then, after appropriately integrating these probabilities over the phase space of the final particles, one would obtain the one-loop probabilities, total or differential with respect either to the emitted photon energy (for nonlinear Compton scattering) or to the produced positron energy (for nonlinear BreitWheeler pair production), to be employed in the codes.

Finally, we comment on the scaling of the radiative corrections due to the vertex correction at $\chi_{0} \gg 1$ in relation with the Ritus-Narozhny conjecture. This should be deduced comparing the leading-order probability amplitude of nonlinear Compton scattering with the first-order amplitude $-i e \Gamma_{s, s^{\prime}, l}\left(p, p^{\prime}, q\right)$, which is obtained starting from the integral over $d^{4} x$ of the matrix element of the components $\quad \Gamma_{R,-}\left(p, p^{\prime}, q ; \phi\right), \quad \Gamma_{R, \perp, j}\left(p, p^{\prime}, q ; \phi\right), \quad$ and $\Gamma_{R, q}\left(p, p^{\prime}, q ; \phi\right)$ between $\bar{U}_{s^{\prime}}\left(p^{\prime}, x\right)$ and $U_{s}(p, x)$ times $\exp (i(q x))$ [see Eqs. (21) and (45) as well as the Appendix for the component $\Gamma_{R, q}\left(p, p^{\prime}, q ; \phi\right)$, which ultimately does not contribute to transition amplitudes]. Now, the integral over $d^{4} x$ pertains to both the leading-order contribution and the first-order correction, and it gives rise to three energy-momentum conservation delta functions and to an overall scaling factor $\chi_{0}^{1 / 3} / \xi_{0}$, corresponding to the formation phase $[12,79]$. The analysis of the matrix elements $\bar{U}_{s^{\prime}}\left(p^{\prime}, x\right)\left[\hat{n}+\Gamma_{R,-}\left(p, p^{\prime}, q ; \phi\right)\right] U_{s}(p, x), \quad \bar{U}_{s^{\prime}}\left(p^{\prime}, x\right)\left[\hat{a}_{j}+\right.$ $\left.\Gamma_{R, \perp, j}\left(p, p^{\prime}, q ; \phi\right)\right] U_{s}(p, x)$, and $\bar{U}_{s^{\prime}}\left(p^{\prime}, x\right)\left[\hat{q}+\Gamma_{R, q}\left(p, p^{\prime}\right.\right.$, $q ; \phi)] U_{s}(p, x)$ is easily carried out in the case of the minus component because one knows that the leading-order amplitude is simply proportional to $\bar{u}_{s^{\prime}}\left(p^{\prime}\right) \hat{n} u_{s}(p)$. Now, the structure of the phase in Eq. (94) shows that at large values of $\chi_{0}$ (due to larger and larger field amplitudes) the main contribution to the integral in $S$ comes from the region $S \lesssim 1 / \chi_{0}^{2 / 3} m^{2}$. Thus, the largest contribution to the radiative corrections comes from the terms in the preexponent in Eq. (95) proportional to $\mathcal{A}_{\perp}^{\prime 2}(\phi) S^{2}=\mathcal{E}_{\perp}^{2}(\phi) S^{2}$. These terms, also taking into account the differential $d S$, give rise to a constant scaling of the radiative corrections with respect to $\chi_{0}$ (possibly times logarithms of $\chi_{0}$ depending on the specific structure of the remaining integrals). One can reach the same conclusion about the components $\Gamma_{R, \perp, j}\left(p, p^{\prime}, q ; \phi\right)$ but the analysis is less straightforward. First, we notice that all the terms in $\Gamma_{R, \perp, j}\left(p, p^{\prime}, q ; \phi\right)$ having the same matrix structure of the leading contribution, i.e., as $\hat{a}_{j}$, feature at most a constant scaling in $\chi_{0}$ (again, apart from possible logarithms of $\chi_{0}$ ). Thus, these terms correspondingly give rise to corrections scaling as $\alpha$ (possibly times logarithms of $\chi_{0}$ ). The remaining terms in Eq. (76) scale at most as $\alpha \chi_{0}^{1 / 3}$ and their matrix structure is always given by products of the matrix $\hat{n}$ times matrices which anticommute with $\hat{n}$. This implies that this scaling also pertains to the corresponding terms in the matrix element of $\bar{U}_{s^{\prime}}\left(p^{\prime}, x\right) \Gamma_{R, \perp, j}\left(p, p^{\prime}, q ; \phi\right) U_{s}(p, x)$ because the fielddependent terms of the Volkov states do not contribute. The conclusion is reached by finally recalling that the leading-order matrix element of nonlinear Compton scattering in a constant crossed field also scales as $\chi_{0}^{1 / 3}[12,79]$, such that the corrections, also in this case, are at most scaling as $\alpha$ possibly times logarithms of $\chi_{0}$. Finally, the results in the last section of the Appendix show that the coefficient $\Gamma_{R, q}\left(p, p^{\prime}, q ; \phi\right)$, instead, features the dominating scaling $\alpha \chi_{0}^{2 / 3}$ arising from the terms proportional to $\mathcal{A}_{\perp}^{\prime 2}(\phi) \sigma=$ $\mathcal{E}_{\perp}^{2}(\phi) \sigma$ [see Eq. (A31)] in agreement with Refs. [78,79], whereas the corresponding leading-order component vanishes due to gauge invariance. Indeed, one can see in Ref. [79] that the scaling as $\alpha \chi_{0}^{2 / 3}$ pertains to terms in $\Gamma^{\mu}\left(p, p^{\prime}, q ; \phi\right)$, which are proportional to $n^{\mu}$ and which then do not contribute to the physical amplitudes. Thus, the 
conclusion is that although the vertex correction provides corrections scaling as $\alpha \chi_{0}^{2 / 3}$ in agreement with the RitusNarozhny conjecture, these corrections stem from terms which do not contribute to physical transition probabilities, at least in the situation under consideration, with the two fermions being on-shell.

\section{CONCLUSIONS}

We have computed the general expression of the oneloop vertex correction in an arbitrary plane-wave background field for the case of two on-shell external electrons and an off-shell external photon. By employing the operator technique within the Furry picture, we have obtained a relatively compact expression, which takes into account exactly the background plane-wave field. By showing explicitly that the vertex correction fulfills a generalized Ward identity, we have singled out the corresponding terms invariant under a gauge transformation of the external photon. As expected, the vertex-correction function features an infrared divergence, which is cured by assigning a small, finite mass to the photon. The ultraviolet divergence of the vertex correction has, instead, been shown to be renormalized as in vacuum.

The important special case of the locally constant field approximation has been studied. We have shown that in the high-field regime $\chi_{0} \gg 1$ the vertex-correction function induces radiative corrections which scale according to the Ritus-Narozhny conjecture as $\alpha \chi_{0}^{2 / 3}$, where $\chi_{0}$ is the amplitude of the quantum nonlinearity parameter. However, the terms precisely scaling as $\alpha \chi_{0}^{2 / 3}$ ultimately do not contribute to physical amplitudes, at least in the case considered in the present paper of on-shell fermions.

\section{ACKNOWLEDGMENTS}

The author would like to thank J. P. Edwards, A. M. Fedotov, S. Meuren, A. A. Mironov, and C. Schubert for insightful discussions. M. A. L. L. gratefully acknowledges the hospitality of the Max Planck Institute for Nuclear Physics in the initial phase of the project. M. A. L. L. is grateful to CONACYT for the financial support during this project. This publication was also supported by the Collaborative Research Centre 1225 funded by Deutsche Forschungsgemeinschaft (DFG, German Research Foundation)—Project No. 273811115—SFB 1225.

\section{APPENDIX: THE COMPONENT $\Gamma_{q}\left(p, p^{\prime}, q ; \phi\right)$ OF THE VERTEX-CORRECTION FUNCTION}

In this appendix, we evaluate more explicitly the component $\Gamma_{q}\left(p, p^{\prime}, q ; \phi\right)$ of the vertex-correction function although we have seen that it does not contribute to any physical transition matrix element.

\section{General structure of $\Gamma_{q}\left(p, p^{\prime}, q ; \phi\right)$}

As we have mentioned in the main text, from the second equality in Eq. (14) and from the definition of $\Gamma^{\mu}\left(p, p^{\prime}, q ; \phi\right)$ in Eq. (21), we obtain

$$
\Gamma_{q}\left(p, p^{\prime}, q ; \phi\right)=-i e^{2} \int \frac{d^{4} k}{(2 \pi)^{4}} \frac{1}{k^{2}-\kappa^{2}+i 0} \gamma^{\lambda} \frac{1}{\hat{\Pi}(\phi)+\hat{k}-\hat{q}-m+i 0} \hat{q} \frac{1}{\hat{\Pi}(\phi)+\hat{k}-m+i 0} \gamma_{\lambda} .
$$

Now, by writing $\hat{q}=\hat{\Pi}(\phi)+\hat{k}-m-[\hat{\Pi}(\phi)+\hat{k}-\hat{q}-m]$ it is clear that we can express $\Gamma_{q}\left(p, p^{\prime}, q ; \phi\right)$ as the difference of two terms containing only one propagator in the plane wave, which significantly simplifies its expression:

$$
\Gamma_{q}\left(p, p^{\prime}, q ; \phi\right)=-i e^{2} \int \frac{d^{4} k}{(2 \pi)^{4}} \frac{1}{k^{2}-\kappa^{2}+i 0} \gamma^{\lambda}\left[\frac{1}{\hat{\Pi}(\phi)+\hat{k}-\hat{q}-m+i 0}-\frac{1}{\hat{\Pi}(\phi)+\hat{k}-m+i 0}\right] \gamma_{\lambda} .
$$

At this point, by following exactly the same steps as in the main text [see Eq. (41)], it is easy to obtain the resulting expression

$$
\begin{aligned}
\Gamma_{q}\left(p, p^{\prime}, q ; \phi\right)= & \frac{\alpha}{4 \pi} \int_{0}^{\infty} \frac{d s d t}{(s+t)^{2}} e^{-i \kappa^{2} t-i \tilde{G}_{s+t}^{2}}\left\{2\left[1-\frac{e \hat{n}\left[\hat{A}\left(\tilde{\psi}_{0, s}\right)-\hat{A}(\phi)\right]}{2 p_{-}^{\prime}}\right]\left[\hat{\pi}_{p^{\prime}}\left(\tilde{\psi}_{0, s}\right)+\frac{\hat{\tilde{G}}_{s}}{s+t}\right]\right. \\
& \left.-\frac{\hat{n}}{(s+t)^{2}} \frac{d \tilde{G}_{s}^{2}}{d k_{-}}+\frac{e \hat{n}\left[\hat{A}\left(\tilde{\psi}_{0, s}\right)-\hat{A}(\phi)\right]}{\tau_{0,-}^{\prime}+k_{-}}\left[\hat{\pi}_{p^{\prime}}\left(\tilde{\psi}_{0, s}\right)-\frac{\tilde{\tilde{G}}_{s}}{s+t}\right]\right\}\left.\right|_{k_{-}=0} \\
& -\frac{\alpha}{4 \pi} \int_{0}^{\infty} \frac{d u d t}{(u+t)^{2}} e^{-i \kappa^{2} t-i \frac{\tilde{G}_{u}^{2}}{u+t}}\left\{2\left[\hat{\pi}_{p}\left(\tilde{\psi}_{0, u}\right)+\frac{\hat{\tilde{G}}_{u}}{u+t}\right]\left[1+\frac{e \hat{n}\left[\hat{A}\left(\tilde{\psi}_{0, u}\right)-\hat{A}(\phi)\right]}{2 p_{-}}\right]\right. \\
& \left.-\frac{\hat{n}}{(u+t)^{2}} \frac{d \tilde{G}_{u}^{2}}{d k_{-}}-\left[\hat{\pi}_{p}\left(\tilde{\psi}_{0, u}\right)-\frac{\hat{\tilde{G}}_{u}}{u+t}\right] \frac{e \hat{n}\left[\hat{A}\left(\tilde{\psi}_{0, u}\right)-\hat{A}(\phi)\right]}{\tau_{0,-}+k_{-}}\right\}\left.\right|_{k_{-}=0},
\end{aligned}
$$


where

$$
\begin{array}{lll}
\tilde{G}_{s}^{\mu}=\int_{0}^{s} d s^{\prime} \pi_{p^{\prime}}^{\mu}\left(\tilde{\psi}_{0, s^{\prime}}\right), & \tilde{\psi}_{0, s}=\phi+2 s \tau_{0,-}^{\prime}+2 s k_{-}, & \tau_{0,-}^{\prime}=\frac{t}{s+t} p_{-}^{\prime}, \\
\tilde{G}_{u}^{\mu}=\int_{0}^{u} d u^{\prime} \pi_{p}^{\mu}\left(\tilde{\psi}_{0, u^{\prime}}\right), & \tilde{\psi}_{0, u}=\phi-2 u \tau_{0,-}-2 u k_{-}, & \tau_{0,-}=\frac{t}{u+t} p_{-} .
\end{array}
$$

Finally, by evaluating the remaining derivatives with respect to $k_{-}$as

$$
\begin{aligned}
& \frac{d \tilde{G}_{s}^{2}}{d k_{-}}=4\left(\tilde{G}_{s} \tilde{G}_{1, s}\right), \\
& \frac{d \tilde{G}_{u}^{2}}{d k_{-}}=4\left(\tilde{G}_{u} \tilde{G}_{1, u}\right),
\end{aligned}
$$

where

$$
\begin{gathered}
\tilde{G}_{1, s}^{\mu}=\int_{0}^{s} d s^{\prime} s^{\prime} \pi_{p^{\prime}}^{\prime \mu}\left(\tilde{\psi}_{0, s^{\prime}}\right)=\frac{1}{2\left(\tau_{0,-}^{\prime}+k_{-}\right)}\left[s \pi_{p^{\prime}}^{\mu}\left(\tilde{\psi}_{0, s}\right)-\int_{0}^{s} d s^{\prime} \pi_{p^{\prime}}^{\mu}\left(\tilde{\psi}_{0, s^{\prime}}\right)\right], \\
\tilde{G}_{1, u}^{\mu}=-\int_{0}^{u} d u^{\prime} u^{\prime} \pi_{p}^{\prime \mu}\left(\tilde{\psi}_{0, u^{\prime}}\right)=\frac{1}{2\left(\tau_{0,-}+k_{-}\right)}\left[u \pi_{p}^{\mu}\left(\tilde{\psi}_{0, u}\right)-\int_{0}^{u} d u^{\prime} \pi_{p}^{\mu}\left(\tilde{\psi}_{0, u^{\prime}}\right)\right],
\end{gathered}
$$

we obtain

$$
\begin{aligned}
\Gamma_{q}\left(p, p^{\prime}, q ; \phi\right)= & \frac{\alpha}{2 \pi} \int_{0}^{\infty} \frac{d s d t}{(s+t)^{2}} e^{-i \kappa^{2} t-i \frac{G_{s}^{2}}{s+t}}\left\{\left(1-\frac{\hat{n} \hat{\Delta}_{0, s}}{2 p_{-}^{\prime}}\right)\left[\hat{\pi}_{p^{\prime}}\left(\psi_{0, s}\right)+\frac{\hat{G}_{s}}{s+t}\right]\right. \\
& \left.-\frac{2 \hat{n}}{(s+t)^{2}}\left(G_{s} G_{1, s}\right)+\frac{\hat{n} \hat{\Delta}_{0, s}}{2 \tau_{0,-}^{\prime}}\left[\hat{\pi}_{p^{\prime}}\left(\psi_{0, s}\right)-\frac{\hat{G}_{s}}{s+t}\right]\right\} \\
& -\frac{\alpha}{2 \pi} \int_{0}^{\infty} \frac{d u d t}{(u+t)^{2}} e^{-i \kappa^{2} t-i \frac{G_{u}^{2}}{u+t}}\left\{\left[\hat{\pi}_{p}\left(\psi_{0, u}\right)+\frac{\hat{G}_{u}}{u+t}\right]\left(1+\frac{\hat{n} \hat{\Delta}_{0, u}}{2 p_{-}}\right)\right. \\
& \left.-\frac{2 \hat{n}}{(u+t)^{2}}\left(G_{u} G_{1, u}\right)-\left[\hat{\pi}_{p}\left(\psi_{0, u}\right)-\frac{\hat{G}_{u}}{u+t}\right] \frac{\hat{n} \hat{\Delta}_{0, u}}{2 \tau_{0,-}}\right\},
\end{aligned}
$$

where

$$
\Delta_{0, s / u}^{\mu}=e\left[A^{\mu}\left(\psi_{0, s / u}\right)-A^{\mu}(\phi)\right]
$$

and where all the quantities without the tilde are the same as those with the tilde but with $k_{-}=0$ :

$$
\begin{aligned}
& G_{s}^{\mu}=\int_{0}^{s} d s^{\prime} \pi_{p^{\prime}}^{\mu}\left(\psi_{0, s^{\prime}}\right), G_{1, s}^{\mu}=\frac{1}{2 \tau_{0,-}^{\prime}}\left[s \pi_{p^{\prime}}^{\mu}\left(\psi_{0, s}\right)-\int_{0}^{s} d s^{\prime} \pi_{p^{\prime}}^{\mu}\left(\psi_{0, s^{\prime}}\right)\right], \quad \psi_{0, s}=\phi+2 s \tau_{0,-}^{\prime}, \\
& G_{u}^{\mu}=\int_{0}^{u} d u^{\prime} \pi_{p}^{\mu}\left(\psi_{0, u^{\prime}}\right), G_{1, u}^{\mu}=\frac{1}{2 \tau_{0,-}}\left[u \pi_{p}^{\mu}\left(\psi_{0, u}\right)-\int_{0}^{u} d u^{\prime} \pi_{p}^{\mu}\left(\psi_{0, u^{\prime}}\right)\right], \quad \psi_{0, u}=\phi-2 u \tau_{0,-} .
\end{aligned}
$$

\section{Regularization of $\Gamma_{q}\left(p, p^{\prime}, q ; \phi\right)$}

Analogous to the other components of the vertex-correction function, the component $\Gamma_{q}\left(p, p^{\prime}, q ; \phi\right)$ has in principle to be regularized as it is apparently logarithmically divergent in the ultraviolet. However, in the case $\Gamma_{R, q}\left(p, p^{\prime}, q ; \phi\right)$, actually, it is not necessary to perform any subtraction of vacuum terms because, as we will show now, it vanishes for $A^{\mu}(\phi)=0$. It is convenient to perform the change of variable $s=x \sigma$ and $t=$ $(1-x) \sigma[u=x \sigma$ and $t=(1-x) \sigma]$ in the first (second) integral in Eq. (A10) [111], and we obtain 


$$
\begin{aligned}
\Gamma_{R, q}\left(p, p^{\prime}, q ; \phi\right)= & \frac{\alpha}{2 \pi} \int_{0}^{\infty} \frac{d \sigma}{\sigma} \int_{0}^{1} d x e^{-i \kappa^{2}(1-x) \sigma} \\
& \times\left\langle e^{-i x^{2} \sigma g_{s}^{2}}\left\{\left(1-\frac{\hat{n} \hat{\Delta}_{0, s}}{2 p_{-}^{\prime}}\right)\left[\hat{\pi}_{p^{\prime}}\left(\theta_{0, \sigma}^{\prime}\right)+x \hat{g}_{s}\right]-2 x^{3} \sigma\left(g_{s} g_{1, s}\right) \hat{n}+\frac{\hat{n} \hat{\Delta}_{0, s}}{2(1-x) p_{-}^{\prime}}\left[\hat{\pi}_{p^{\prime}}\left(\theta_{0, \sigma}^{\prime}\right)-x \hat{g}_{s}\right]\right\}\right. \\
& \left.-e^{-i x^{2} \sigma g_{u}^{2}}\left\{\left[\hat{\pi}_{p}\left(\theta_{0, \sigma}\right)+x \hat{g}_{u}\right]\left(1+\frac{\hat{n} \hat{\Delta}_{0, u}}{2 p_{-}}\right)-2 x^{3} \sigma\left(g_{u} g_{1, u}\right) \hat{n}-\left[\hat{\pi}_{p}\left(\theta_{0, \sigma}\right)-x \hat{g}_{u}\right] \frac{\hat{n} \hat{\Delta}_{0, u}}{2(1-x) p_{-}}\right\}\right\rangle,
\end{aligned}
$$

where

$$
\begin{aligned}
& \theta_{0, \sigma}^{\prime}=\phi+2 x(1-x) p_{-}^{\prime} \sigma, \\
& \theta_{0, \sigma}=\phi-2 x(1-x) p_{-} \sigma,
\end{aligned}
$$

and

$$
\begin{gathered}
g_{s}^{\mu}=\frac{G_{s}^{\mu}}{s}=\int_{0}^{1} d \eta \pi_{p^{\prime}}^{\mu}\left(\theta_{0, \eta \sigma}^{\prime}\right), g_{1, s}^{\mu}=\frac{G_{1, s}^{\mu}}{s^{2}}=\frac{1}{2 x(1-x) \sigma p_{-}^{\prime}}\left[\pi_{p^{\prime}}^{\mu}\left(\theta_{0, \sigma}^{\prime}\right)-\int_{0}^{1} d \eta \pi_{p^{\prime}}^{\mu}\left(\theta_{0, \eta \sigma}^{\prime}\right)\right], \\
g_{u}^{\mu}=\frac{G_{u}^{\mu}}{u}=\int_{0}^{1} d \eta \pi_{p}^{\mu}\left(\theta_{0, \eta \sigma}\right), g_{1, u}^{\mu}=\frac{G_{1, u}^{\mu}}{u^{2}}=\frac{1}{2 x(1-x) \sigma p_{-}}\left[\pi_{p}^{\mu}\left(\theta_{0, \sigma}\right)-\int_{0}^{1} d \eta \pi_{p}^{\mu}\left(\theta_{0, \eta \sigma}\right)\right],
\end{gathered}
$$

and where $\Delta_{0, s}^{\mu}$ and $\Delta_{0, u}^{\mu}$ are intended to be expressed in terms of the new variables as

$$
\begin{gathered}
\Delta_{0, s}^{\mu}=\mathcal{A}^{\mu}\left(\theta_{0, \sigma}^{\prime}\right)-\mathcal{A}^{\mu}(\phi), \\
\Delta_{0, u}^{\mu}=\mathcal{A}^{\mu}\left(\theta_{0, \sigma}\right)-\mathcal{A}^{\mu}(\phi),
\end{gathered}
$$

with $\mathcal{A}^{\mu}(\phi)=e A^{\mu}(\phi)$. Note that $g_{1, s}^{\mu}$ and $g_{1, u}^{\mu}$ tend to constant values for $\sigma \rightarrow 0$. Since $\Delta_{0, s}^{\mu}$ and $\Delta_{0, u}^{\mu}$ vanish at $\sigma=0$, the only problematic terms are those in the square brackets in Eq. (A14) containing exclusively $\hat{\pi}_{p^{\prime}}\left(\theta_{0, \sigma}^{\prime}\right)+x \hat{g}_{s}$ and $\hat{\pi}_{p}\left(\theta_{0, \sigma}\right)+x \hat{g}_{u}$. However, since $\Gamma_{q}\left(p, p^{\prime}, q ; \phi\right)$ will finally be multiplied by Volkov states both from the left and on the right, we can replace $\hat{\pi}_{p^{\prime}}\left(\theta_{0, \sigma}^{\prime}\right)+x \hat{g}_{s}$ and $\hat{\pi}_{p}\left(\theta_{0, \sigma}\right)+x \hat{g}_{u}$ in the terms which do not contain other gamma matrices as

$$
\begin{aligned}
& \hat{\pi}_{p^{\prime}}\left(\theta_{0, \sigma}^{\prime}\right)+x \hat{g}_{s} \rightarrow m(1+x)+\hat{\pi}_{p^{\prime}}\left(\theta_{0, \sigma}^{\prime}\right)-\hat{\pi}_{p^{\prime}}(\phi)+x \int_{0}^{1} d \eta\left[\hat{\pi}_{p^{\prime}}\left(\theta_{0, \eta \sigma}^{\prime}\right)-\hat{\pi}_{p^{\prime}}(\phi)\right], \\
& \hat{\pi}_{p}\left(\theta_{0, \sigma}\right)+x \hat{g}_{u} \rightarrow m(1+x)+\hat{\pi}_{p}\left(\theta_{0, \sigma}\right)-\hat{\pi}_{p}(\phi)+x \int_{0}^{1} d \eta\left[\hat{\pi}_{p}\left(\theta_{0, \eta \sigma}\right)-\hat{\pi}_{p}(\phi)\right] .
\end{aligned}
$$

In this way, the only remaining divergent terms are those proportional to $m(1+x)$, but these divergences cancel each other in Eq. (A14), which can conveniently be written in the manifestly convergent form

$$
\begin{aligned}
\Gamma_{R, q}\left(p, p^{\prime}, q ; \phi\right)= & \frac{\alpha}{2 \pi} \int_{0}^{\infty} \frac{d \sigma}{\sigma} \int_{0}^{1} d x e^{-i \kappa^{2}(1-x) \sigma}\left\langle m(1+x)\left(e^{-i x^{2} \sigma g_{s}^{2}}-e^{-i x^{2} \sigma g_{u}^{2}}\right)\right. \\
& +e^{-i x^{2} \sigma g_{s}^{2}}\left\{\hat{\pi}_{p^{\prime}}\left(\theta_{0, \sigma}^{\prime}\right)-\hat{\pi}_{p^{\prime}}(\phi)+x \int_{0}^{1} d \eta\left[\hat{\pi}_{p^{\prime}}\left(\theta_{0, \eta \sigma}^{\prime}\right)-\hat{\pi}_{p^{\prime}}(\phi)\right]\right. \\
& \left.+\frac{x}{1-x} \frac{\hat{n} \hat{\Delta}_{0, s} \hat{\pi}_{p^{\prime}}\left(\theta_{0, \sigma}^{\prime}\right)}{2 p_{-}^{\prime}}-\frac{x(2-x)}{1-x} \frac{\hat{n} \hat{\Delta}_{0, s} \hat{g}_{s}}{2 p_{-}^{\prime}}-2 x^{3} \sigma\left(g_{s} g_{1, s}\right) \hat{n}\right\} \\
& -e^{-i x^{2} \sigma g_{u}^{2}}\left\{\hat{\pi}_{p}\left(\theta_{0, \sigma}\right)-\hat{\pi}_{p}(\phi)+x \int_{0}^{1} d \eta\left[\hat{\pi}_{p}\left(\theta_{0, \eta \sigma}\right)-\hat{\pi}_{p}(\phi)\right]\right. \\
& \left.\left.-\frac{x}{1-x} \frac{\hat{\pi}_{p}\left(\theta_{0, \sigma}\right) \hat{n} \hat{\Delta}_{0, u}}{2 p_{-}}+\frac{x(2-x)}{1-x} \frac{\hat{g}_{u} \hat{n} \hat{\Delta}_{0, u}}{2 p_{-}}-2 x^{3} \sigma\left(g_{u} g_{1, u}\right) \hat{n}\right\}\right\rangle .
\end{aligned}
$$


This expression also shows that $\Gamma_{R, q}\left(p, p^{\prime}, q ; \phi\right)$ tends to zero for $A^{\mu}(\phi) \rightarrow 0$.

\section{The locally constant field approximation}

As we have mentioned in the main text, in order to study the structure of the component $\Gamma_{R, q}^{\mu}\left(p, p^{\prime}, q ; \phi\right)$ of the vertex-correction function within the LCFA, it is useful to rewrite the phases $G_{s}^{2} /(s+t)$ and $G_{u}^{2} /(u+t)$ in a convenient form [see Eq. (A10)]. Although, as we have seen in the main text, the component $\Gamma_{R, q}\left(p, p^{\prime}, q ; \phi\right)$ does not contribute to any transition matrix element, it is useful to compute the corresponding expression within the LCFA. By starting from the identity $v^{2}=2 v_{+} v_{-}-v_{\perp}^{2}$, valid for a generic four-vector $v^{\mu}$, it can easily be shown that

$$
\begin{aligned}
& G_{s}^{2}=s^{2}\left(m^{2}+\delta m_{0, s}^{2}\right), \\
& G_{u}^{2}=u^{2}\left(m^{2}+\delta m_{0, u}^{2}\right),
\end{aligned}
$$

where we have introduced the laser-induced square mass corrections

$$
\begin{gathered}
\delta m_{0, s}^{2}=\frac{1}{s} \int_{0}^{s} d s^{\prime} \mathcal{A}_{\perp}^{2}\left(\psi_{0, s^{\prime}}\right)-\frac{1}{s^{2}}\left[\int_{0}^{s} d s^{\prime} \mathcal{A}_{\perp}\left(\psi_{0, s^{\prime}}\right)\right]^{2}=\frac{1}{s} \int_{0}^{s} d s^{\prime} \boldsymbol{\Delta}_{0, s^{\prime}, \perp}^{2}-\frac{1}{s^{2}}\left(\int_{0}^{s} d s^{\prime} \boldsymbol{\Delta}_{0, s^{\prime}, \perp}\right)^{2}, \\
\delta m_{0, u}^{2}=\frac{1}{u} \int_{0}^{u} d u^{\prime} \mathcal{A}_{\perp}^{2}\left(\psi_{0, u^{\prime}}\right)-\frac{1}{u^{2}}\left[\int_{0}^{u} d u^{\prime} \mathcal{A}_{\perp}\left(\psi_{0, u^{\prime}}\right)\right]^{2}=\frac{1}{u} \int_{0}^{u} d u^{\prime} \boldsymbol{\Delta}_{0, u^{\prime}, \perp}^{2}-\frac{1}{u^{2}}\left(\int_{0}^{u} d u^{\prime} \boldsymbol{\Delta}_{0, u^{\prime}, \perp}\right)^{2} .
\end{gathered}
$$

We notice that for the present case of on-shell electrons, the quantities $G_{s}^{2}$ and $G_{u}^{2}$ are non-negative. By proceeding as in the main text we arrive to the approximated expressions

$$
\delta m_{0, s}^{2} \approx \frac{1}{3} m^{2}\left(\frac{t}{s+t}\right)^{2} m^{4} s^{2} \chi_{p^{\prime}}^{2}(\phi), \quad \delta m_{0, u}^{2} \approx \frac{1}{3} m^{2}\left(\frac{t}{u+t}\right)^{2} m^{4} u^{2} \chi_{p}^{2}(\phi),
$$

where $\chi_{p}(\phi)=p_{-}\left|\mathcal{E}_{\perp}(\phi)\right| / m^{3}$ and $\chi_{p^{\prime}}(\phi)=p_{-}^{\prime}\left|\mathcal{E}_{\perp}(\phi)\right| / m^{3}$, with $\mathcal{E}_{\perp}(\phi)=-\mathcal{A}_{\perp}^{\prime}(\phi)$. These approximated expressions are valid if $\eta_{0} / \chi_{0}^{2 / 3}=\chi_{0}^{1 / 3} / \xi_{0} \ll 1$, together with the original assumption about the product of the laser electric field amplitude and the laser wavelength being large, which corresponds to the condition $\xi_{0} \gg 1$, as discussed at the beginning of Sec. VI $[45,48,49,68,89,90,98,99,101]$. The final expressions of the phases $G_{s}^{2} /(s+t)=x^{2} \sigma g_{s}^{2}$ and $G_{u}^{2} /(u+t)=x^{2} \sigma g_{u}^{2}$ within the LCFA are

$$
\begin{aligned}
& x^{2} \sigma g_{s}^{2}=x^{2} m^{2} \sigma\left[1+\frac{1}{3} x^{2}(1-x)^{2} m^{4} \sigma^{2} \chi_{p^{\prime}}^{2}(\phi)\right], \\
& x^{2} \sigma g_{u}^{2}=x^{2} m^{2} \sigma\left[1+\frac{1}{3} x^{2}(1-x)^{2} m^{4} \sigma^{2} \chi_{p}^{2}(\phi)\right] .
\end{aligned}
$$

We pass now to the preexponential functions, and it is easy to perform the corresponding expansions. Having in mind that the matrix element of $\Gamma_{R, q}\left(p, p^{\prime}, q ; \phi\right)$ between $\bar{U}_{s^{\prime}}\left(p^{\prime}, x\right)$ and $U_{s}(p, x)$ will ultimately be needed and starting from the expression in Eq. (A23) of $\Gamma_{R, q}\left(p, p^{\prime}, q\right.$; $\left.\phi\right)$, we obtain

$$
\begin{aligned}
\Gamma_{R, q}\left(p, p^{\prime}, q ; \phi\right)= & \frac{\alpha}{2 \pi} \int_{0}^{\infty} \frac{d \sigma}{\sigma} \int_{0}^{1} d x e^{-i \kappa^{2}(1-x) \sigma}\left\langle m(1+x)\left(e^{-i x^{2} \sigma g_{s}^{2}}-e^{-i x^{2} \sigma g_{u}^{2}}\right)\right. \\
& +e^{-i x^{2} \sigma g_{s}^{2}} \frac{b_{0}^{\prime}}{p_{-}^{\prime}}\left\{\hat{n} \hat{\mathcal{A}}^{\prime}\left[\frac{x-2}{2} p_{-}^{\prime} \gamma_{\perp} \cdot \mathcal{V}_{\perp}-\left(x-\frac{x-2}{2} \frac{q_{-}}{p_{-}}\right) m\right]-\frac{2}{3} \frac{2 x-3}{1-x} b_{0}^{\prime} \mathcal{A}_{\perp}^{\prime 2} \hat{n}\right\} \\
& \left.+e^{-i x^{2} \sigma g_{u}^{2}} \frac{b_{0}}{p_{-}}\left\{\left[\frac{x-2}{2} p_{-} \gamma_{\perp} \cdot \mathcal{V}_{\perp}+\left(x+\frac{x-2}{2} \frac{q-}{p_{-}^{\prime}}\right) m\right] \hat{n} \hat{\mathcal{A}}^{\prime}+\frac{2}{3} \frac{2 x-3}{1-x} b_{0} \mathcal{A}_{\perp}^{\prime 2} \hat{n}\right\}\right\rangle,
\end{aligned}
$$

where $b_{0}=x(1-x) p_{-} \sigma$ and $b_{0}^{\prime}=x(1-x) p_{-}^{\prime} \sigma$, and where $\mathcal{V}_{\perp}=\mathcal{V}_{\perp}(\phi)$ is defined in Eq. (91). As we have done in the main text for the components $\Gamma_{R,-}\left(p, p^{\prime}, q ; \phi\right)$ and $\Gamma_{R, \perp, j}\left(p, p^{\prime}, q ; \phi\right)$, it is interesting to comment on the scaling of $\Gamma_{R, q}\left(p, p^{\prime}, q ; \phi\right)$ at $\chi_{0} \gg 1$. Now, the structure of the phases in Eqs. (A29) and (A30) shows that at large 
values of $\chi_{0}$ the main contribution to the integral in $\sigma$ comes from the region $\sigma \lesssim 1 / \chi_{0}^{2 / 3} \mathrm{~m}^{2}$. Thus, the terms in the preexponent in Eq. (A31) proportional to $\mathcal{A}_{\perp}^{\prime 2}(\phi) \sigma=$ $\mathcal{E}_{\perp}^{2}(\phi) \sigma$ give rise to the scaling $\alpha \chi_{0}^{2 / 3}$ of the radiative corrections in agreement with the results in Refs. [78,79] (in particular, in Ref. [79] one can verify that the terms scaling as $\alpha \chi_{0}^{2 / 3}$ feature the matrix structure corresponding to $\hat{n}$ here).
[1] D. Hanneke, S. Fogwell, and G. Gabrielse, Phys. Rev. Lett. 100, 120801 (2008).

[2] S. Sturm, A. Wagner, B. Schabinger, J. Zatorski, Z. Harman, W. Quint, G. Werth, C. H. Keitel, and K. Blaum, Phys. Rev. Lett. 107, 023002 (2011).

[3] V. B. Berestetskii, E. M. Lifshitz, and L. P. Pitaevskii, Quantum Electrodynamics (Elsevier ButterworthHeinemann, Oxford, 1982).

[4] E. S. Fradkin, D. M. Gitman, and Sh. M. Shvartsman, Quantum Electrodynamics with Unstable Vacuum (Springer, Berlin, 1991).

[5] W. Dittrich and M. Reuter, Effective Lagrangians in Quantum Electrodynamics (Springer, Heidelberg, 1985).

[6] J. W. Yoon, C. Jeon, J. Shin, S. K. Lee, H. W. Lee, I. W. Choi, H. T. Kim, J. H. Sung, and C. H. Nam, Opt. Express 27, 20412 (2019).

[7] D. Papadopoulos, J. Zou, C. Le Blanc, G. Chériaux, P. Georges, F. Druon, G. Mennerat, P. Ramirez, L. Martin, A. Fréneaux, A. Beluze, N. Lebas, P. Monot, F. Mathieu, and P. Audebert, High Power Laser Sci. Eng. 4, e34 (2016).

[8] Extreme Light Infrastructure (ELI), https://eli-laser.eu/.

[9] Center for Relativistic Laser Science (CoReLS), https:// www.ibs.re.kr/eng/sub02_03_05.do.

[10] Exawatt Center for Extreme Light Studies (XCELS), http://www.xcels.iapras.ru/.

[11] H. Mitter, Acta Phys. Austriaca XIV, 397 (1975).

[12] V. I. Ritus, J. Sov. Laser Res. 6, 497 (1985).

[13] F. Ehlotzky, K. Krajewska, and J. Z. Kamiński, Rep. Prog. Phys. 72, 046401 (2009).

[14] H. R. Reiss, Eur. Phys. J. D 55, 365 (2009).

[15] A. Di Piazza, C. Müller, K. Z. Hatsagortsyan, and C. H. Keitel, Rev. Mod. Phys. 84, 1177 (2012).

[16] G. V. Dunne, Eur. Phys. J. Special Topics 223, 1055 (2014).

[17] C. Bula, K. T. McDonald, E. J. Prebys, C. Bamber, S. Boege, T. Kotseroglou, A. C. Melissinos, D. D. Meyerhofer, W. Ragg, D. L. Burke, R. C. Field, G. Horton-Smith, A. C. Odian, J. E. Spencer, D. Walz, S. C. Berridge, W. M. Bugg, K. Shmakov, and A. W. Weidemann, Phys. Rev. Lett. 76, 3116 (1996).

[18] D. L. Burke, R. C. Field, G. Horton-Smith, J. E. Spencer, D. Walz, S. C. Berridge, W. M. Bugg, K. Shmakov, A. W. Weidemann, C. Bula, K. T. McDonald, E. J. Prebys, C. Bamber, S. J. Boege, T. Koffas, T. Kotseroglou, A. C. Melissinos, D. D. Meyerhofer, D. A. Reis, and W. Ragg, Phys. Rev. Lett. 79, 1626 (1997).

[19] C. Bamber, S. J. Boege, T. Koffas, T. Kotseroglou, A. C. Melissinos, D. D. Meyerhofer, D. A. Reis, W. Ragg, C. Bula, K. T. McDonald, E. J. Prebys, D. L. Burke,
R. C. Field, G. Horton-Smith, J. E. Spencer, D. Walz, S. C. Berridge, W. M. Bugg, K. Shmakov, and A. W. Weidemann, Phys. Rev. D 60, 092004 (1999).

[20] J. M. Cole et al., Phys. Rev. X 8, 011020 (2018).

[21] K. Poder et al., Phys. Rev. X 8, 031004 (2018).

[22] I. I. Gol'dman, Phys. Lett. 8, 103 (1964).

[23] A. I. Nikishov and V. I. Ritus, Sov. Phys. JETP 19, 529 (1964).

[24] V. N. Baier, V. M. Katkov, and V. M. Strakhovenko, Electromagnetic Processes at High Energies in Oriented Single Crystals (World Scientific, Singapore, 1998).

[25] D. Yu. Ivanov, G. L. Kotkin, and V. G. Serbo, Eur. Phys. J. C 36, 127 (2004).

[26] M. Boca and V. Florescu, Phys. Rev. A 80, 053403 (2009).

[27] C. Harvey, T. Heinzl, and A. Ilderton, Phys. Rev. A 79, 063407 (2009).

[28] F. Mackenroth, A. Di Piazza, and C. H. Keitel, Phys. Rev. Lett. 105, 063903 (2010).

[29] M. Boca and V. Florescu, Eur. Phys. J. D 61, 449 (2011).

[30] F. Mackenroth and A. Di Piazza, Phys. Rev. A 83, 032106 (2011).

[31] D. Seipt and B. Kämpfer, Phys. Rev. A 83, 022101 (2011).

[32] D. Seipt and B. Kämpfer, Phys. Rev. ST Accel. Beams 14, 040704 (2011).

[33] V. Dinu, T. Heinzl, and A. Ilderton, Phys. Rev. D 86, 085037 (2012).

[34] K. Krajewska and J. Z. Kamiński, Phys. Rev. A 85, 062102 (2012).

[35] V. Dinu, Phys. Rev. A 87, 052101 (2013).

[36] D. Seipt and B. Kämpfer, Phys. Rev. A 88, 012127 (2013).

[37] K. Krajewska, M. Twardy, and J. Z. Kamiński, Phys. Rev. A 89, 032125 (2014).

[38] T. N. Wistisen, Phys. Rev. D 90, 125008 (2014).

[39] C. N. Harvey, A. Ilderton, and B. King, Phys. Rev. A 91, 013822 (2015).

[40] D. Seipt, V. Kharin, S. Rykovanov, A. Surzhykov, and S. Fritzsche, J. Plasma Phys. 82, 655820203 (2016).

[41] D. Seipt, A. Surzhykov, S. Fritzsche, and B. Kämpfer, New J. Phys. 18, 023044 (2016).

[42] A. Angioi, F. Mackenroth, and A. Di Piazza, Phys. Rev. A 93, 052102 (2016).

[43] C. N. Harvey, A. Gonoskov, M. Marklund, and E. Wallin, Phys. Rev. A 93, 022112 (2016).

[44] A. Angioi and A. Di Piazza, Phys. Rev. Lett. 121, 010402 (2018).

[45] A. Di Piazza, M. Tamburini, S. Meuren, and C. H. Keitel, Phys. Rev. A 98, 012134 (2018).

[46] V. Dinu and G. Torgrimsson, Phys. Rev. D 97, 036021 (2018). 
[47] I. A. Aleksandrov, G. Plunien, and V. M. Shabaev, Phys. Rev. D 99, 016020 (2019).

[48] A. Di Piazza, M. Tamburini, S. Meuren, and C. H. Keitel, Phys. Rev. A 99, 022125 (2019).

[49] A. Ilderton, B. King, and D. Seipt, Phys. Rev. A 99, 042121 (2019).

[50] H. R. Reiss, J. Math. Phys. (N.Y.) 3, 59 (1962).

[51] N. B. Narozhny and M. S. Fofanov, J. Exp. Theor. Phys. 90, 415 (2000).

[52] S. P. Roshchupkin, Phys. At. Nucl. 64, 243 (2001).

[53] T. Heinzl, A. Ilderton, and M. Marklund, Phys. Lett. B 692, 250 (2010).

[54] T.-O. Müller and C. Müller, Phys. Lett. B 696, 201 (2011).

[55] A. I. Titov, H. Takabe, B. Kämpfer, and A. Hosaka, Phys. Rev. Lett. 108, 240406 (2012).

[56] T. Nousch, D. Seipt, B. Kämpfer, and A. Titov, Phys. Lett. B 715, 246 (2012).

[57] K. Krajewska, C. Müller, and J. Z. Kamiński, Phys. Rev. A 87, 062107 (2013).

[58] M. J. A. Jansen and C. Müller, Phys. Rev. A 88, 052125 (2013).

[59] S. Augustin and C. Müller, Phys. Lett. B 737, 114 (2014).

[60] S. Meuren, K. Z. Hatsagortsyan, C. H. Keitel, and A. Di Piazza, Phys. Rev. D 91, 013009 (2015).

[61] S. Meuren, C. H. Keitel, and A. Di Piazza, Phys. Rev. D 93, 085028 (2016).

[62] B. King, Phys. Rev. A 101, 042508 (2020).

[63] W. H. Furry, Phys. Rev. 81, 115 (1951).

[64] D. M. Volkov, Z. Phys. 94, 250 (1935).

[65] J. Schwinger, Phys. Rev. 82, 664 (1951).

[66] V. N. Baier, V. M. Katkov, A. I. Milstein, and V. M. Strakhovenko, Sov. Phys. JETP 42, 400 (1976).

[67] V. N. Baier, A. I. Milstein, and V. M. Strakhovenko, Sov. Phys. JETP 42, 961 (1976).

[68] A. Di Piazza, A. I. Milstein, and C. H. Keitel, Phys. Rev. A 76, 032103 (2007).

[69] A. Di Piazza and A. I. Milstein, Phys. Rev. A 77, 042102 (2008).

[70] A. Di Piazza, Ann. Phys. (N.Y.) 338, 302 (2013).

[71] A. Di Piazza, Phys. Rev. D 97, 056028 (2018).

[72] V. I. Ritus, Sov. Phys. JETP 30, 1181 (1970).

[73] V. I. Ritus, Ann. Phys. (N.Y.) 69, 555 (1972).

[74] V. I. Ritus, Nucl. Phys. B44, 236 (1972).

[75] D. A. Morozov and V. I. Ritus, Nucl. Phys. B86, 309 (1975).

[76] N. B. Narozhny, Phys. Rev. D 20, 1313 (1979).

[77] N. B. Narozhny, Phys. Rev. D 21, 1176 (1980).

[78] D. A. Morozov, N. B. Narozhny, and V. I. Ritus, Sov. Phys. JETP 53, 1103 (1981).

[79] D. A. Morozov, N. B. Narozhny, and V. I. Ritus, Fiz. Inst. Akad. Nauk 84, 1 (1981).

[80] A. A. Mironov, S. Meuren, and A. M. Fedotov, Phys. Rev. D 102, 053005 (2020).

[81] E. K. Akhmedov, Sov. Phys. JETP 58, 883 (1983).

[82] E. K. Akhmedov, Phys. At. Nucl. 74, 1299 (2011).

[83] A. M. Fedotov, J. Phys. Conf. Ser. 826, 012027 (2017).

[84] W. Becker and H. Mitter, J. Phys. A 8, 1638 (1975).

[85] S. Meuren, C. H. Keitel, and A. Di Piazza, Phys. Rev. D 88, 013007 (2013).

[86] J. M. Jauch and F. Rohrlich, The Theory of Photons and Electrons (Springer, Berlin, 1976).
[87] C. Itzykson and J.-B. Zuber, Quantum Field Theory (McGraw-Hill Inc., New York, 1980).

[88] M. Schwartz, Quantum Field Theory and the Standard Model (Cambridge University Press, Cambridge, England, 2014).

[89] T. Podszus and A. Di Piazza, Phys. Rev. D 99, 076004 (2019).

[90] A. Ilderton, Phys. Rev. D 99, 085002 (2019).

[91] M. E. Peskin and D. V. Schroeder, An Introduction to Quantum Field Theory (Westview Press, Boulder, 1995).

[92] The terms with three gamma matrices can be further reduced according to the identity

$$
\begin{aligned}
\hat{A} \hat{B} \hat{C} & =\frac{1}{4} \operatorname{tr}\left(\gamma_{\mu} \hat{A} \hat{B} \hat{C}\right) \gamma^{\mu}-\frac{1}{4} \operatorname{tr}\left(\gamma^{5} \gamma_{\mu} \hat{A} \hat{B} \hat{C}\right) \gamma^{5} \gamma^{\mu} \\
& =\hat{A}(B C)-\hat{B}(A C)+\hat{C}(A B)+i \varepsilon_{\mu \nu \lambda \rho} \gamma^{5} \gamma^{\mu} A^{\nu} B^{\lambda} C^{\rho},
\end{aligned}
$$

where $\gamma^{5}=i \gamma^{0} \gamma^{1} \gamma^{2} \gamma^{3}$ and $\varepsilon^{\mu \nu \lambda \rho}$ is the completely antisymmetric tensor with $\varepsilon^{0123}=+1$, which is valid for three arbitrary four-vectors $A^{\mu}, B^{\mu}$, and $C^{\mu}$.

[93] C. Schubert, Phys. Rep. 355, 73 (2001).

[94] J. Schwinger, Phys. Rev. 73, 416 (1948).

[95] V. N. Baier, V. M. Katkov, and V. M. Strakhovenko, Dokl. Akad. Nauk SSSR 197, 66 (1971).

[96] S. Meuren and A. Di Piazza, Phys. Rev. Lett. 107, 260401 (2011).

[97] A. Ilderton, B. King, and S. Tang, arXiv:2008.08578.

[98] V. N. Baier, V. M. Katkov, and V. M. Strakhovenko, Nucl. Phys. B328, 387 (1989).

[99] M. K. Khokonov and H. Nitta, Phys. Rev. Lett. 89, 094801 (2002).

[100] T. N. Wistisen, Phys. Rev. D 92, 045045 (2015).

[101] V. Dinu, C. Harvey, A. Ilderton, M. Marklund, and G. Torgrimsson, Phys. Rev. Lett. 116, 044801 (2016).

[102] E. Raicher, Q. Z. Lv, C. H. Keitel, and K. Z. Hatsagortsyan, arXiv:2003.06217.

[103] S. Meuren, K. Z. Hatsagortsyan, C. H. Keitel, and A. Di Piazza, Phys. Rev. Lett. 114, 143201 (2015).

[104] A. R. Bell and J. G. Kirk, Phys. Rev. Lett. 101, 200403 (2008).

[105] N. V. Elkina, A. M. Fedotov, I. Yu. Kostyukov, M. V. Legkov, N. B. Narozhny, E. N. Nerush, and H. Ruhl, Phys. Rev. ST Accel. Beams 14, 054401 (2011).

[106] E. N. Nerush, I. Yu. Kostyukov, A. M. Fedotov, N. B. Narozhny, N. V. Elkina, and H. Ruhl, Phys. Rev. Lett. 106, 035001 (2011).

[107] C. P. Ridgers, J. Kirk, R. Duclous, T. Blackburn, C. Brady, K. Bennett, T. D. Arber, and A. R. Bell, J. Comput. Phys. 260, 273 (2014).

[108] A. Gonoskov, S. Bastrakov, E. Efimenko, A. Ilderton, M. Marklund, I. Meyerov, A. Muraviev, A. Sergeev, I. Surmin, and E. Wallin, Phys. Rev. E 92, 023305 (2015).

[109] E. G. Gelfer, A. A. Mironov, A. M. Fedotov, V. F. Bashmakov, E. N. Nerush, I. Y. Kostyukov, and N. B. Narozhny, Phys. Rev. A 92, 022113 (2015).

[110] M. Tamburini, A. Di Piazza, and C. H. Keitel, Sci. Rep. 7, 5694 (2017).

[111] Note that in this section and afterwards the symbol $x$ should not be confused with a spacetime point as it indicates a single, real variable. 